Mathématiques et sciences humaines
Mathematics and social sciences

151 | Automne 2000

Varia

\title{
Moyenne selon une loi de composition
}

Mean according to a composition law

\section{Charles Antoine}

\section{OpenEdition}

Journals

Édition électronique

URL : http://journals.openedition.org/msh/2816

DOI : $10.4000 /$ msh.2816

ISSN : 1950-6821

\section{Éditeur}

Centre d'analyse et de mathématique sociales de l'EHESS

\section{Édition imprimée}

Date de publication : 1 septembre 2000

ISSN : 0987-6936

\section{Référence électronique}

Charles Antoine, " Moyenne selon une loi de composition », Mathématiques et sciences humaines [En ligne], 151 | Automne 2000, mis en ligne le 10 février 2006, consulté le 23 juillet 2020. URL : http:// journals.openedition.org/msh/2816 ; DOI : https://doi.org/10.4000/msh.2816 
Math. \& Sci. hum., (38 année, $\mathrm{n}^{\circ} 151,2000$, p. 5-35)

\title{
MOYENNE SELON UNE LOI DE COMPOSITION
}

\author{
Charles ANTOINE
}

RÉSUMÉ - Cet article présente un modèle algébrique du concept de moyenne, dans le cas discret et fini, mais non nécessairement restreint au champ numérique. La démarche adoptée est purement formelle et axiomatique bien qu'elle s'inspire, naturellement, des modèles empiriques que sont les moyennes pythagoriciennes (les classiques moyennes d'ordre $r$ ).

Dans la perspective d'éventuelles applications dans le domaine des sciences de l'homme, on a cherché à s'affranchir des données strictement numériques, sans pour autant les exclure. Aussi, avons-nous défini une opération susceptible d'incarner l'idée de moyenne, en minimisant l'outillage mathématique nécessaire à sa mise en æuvre. En effet, comme point d'ancrage à sa construction, nous n'avons fait intervenir que deux notions : celle d'ordre de treillis et celle de loi de composition. La première permet de formaliser l'idée de valeur intermédiaire, la seconde celle d'agrégation selon le critère de la valeur constante ; deux principes dont nous pensons qu'ils constituent le fondement même de toute théorie des moyennes.

On commence par étudier les moyennes opérant sur deux objets seulement, dont on dégage quelques propriétés élémentaires avant d'examiner les conditions d'obtention. Ensuite on généralise à plus de deux objets en signalant les propriétés barycentriques et de pondération. Compatibilité.

MOTS-CLÉS! - Treillis, Isomorphisme d’ordre, Magma, Application n-aire, Loi idempotente,

SUMMARY - Mean according to a composition law.

This article presents an algebraic model of the concept of mean, in the discontinuous and finite case, but not necessarily restricted to numerical calculation. The approach which has been used is strictly formal and axiomatic even though it is, of course, issued from empirical models i.e. Pythagorean means (type r classical means).

In view of possible applications in the human sciences, there has been an attempt to break away from strict numerical data, without excluding them entirely. We have determined a process which is able to embody the concept of average, by minimizing the mathematical tools needed for its application. As a starting point for its construction, we have used only two notions, those of latice, and of composition law. The first notion enables us to formalize the idea of intermediate value and the second the idea of aggregation according to the criterion of constant; we think that these two principles make up the very foundation of the theory of averages.

We begin by studying the means which operate on two objects only. We set out a few elementary characteristics before proceeding with an examination of the obtention conditions. We then generalize to more than two objects by pointing out the barycentric characteristics and weighted means.

KEYWORDS - Lattice, Order isomorphism, Magma, n-ary application, Idempotence law, Compatibility.

Article communiqué par B. Leclerc.

${ }^{1}$ Professeur de mathématiques au lycée Roger Verlomme à Paris. 


\section{INTRODUCTION}

On se propose de présenter un modèle algébrique de l'opération moyenne. L'objectif de cette construction, qui se veut axiomatique, est double. D'une part, on cherche à dégager un principe unificateur, susceptible de regrouper la variété des expressions algébriques qui revendiquent le statut de moyenne ; et d'autre part, dans la perspective d'éventuelles applications dans le domaine des sciences de l'homme, on souhaite pouvoir étendre la notion en dehors du champ numérique.

Aussi, avons-nous cherché à définir une opération susceptible d'incarner l'idée de moyenne, en minimisant l'outillage mathématique nécessaire à sa mise en œuvre. Il nous a semblé que les deux notions!: ordre de treillis et loi de composition, constituaient le minimum requis. En effet, la première permet de formaliser l'idée de valeur intermédiaire, la seconde celle d'agrégation selon le critère de la valeur constante (invariance de la composition de données variables par substitution d'une valeur constante) ; deux principes dont nous pensons qu'ils constituent le fondement même de toute théorie des moyennes.

Le présent article se limite à l'étude du dénombrable fini - on parle, alors, de moyennes discrètes -, mais il n'est pas exclu, moyennant une théorie de la mesure ad hoc, que l'on puisse adapter le prototype aux structures continues, à l'instar de la moyenne arithmétique.

La première partie de cet exposé se concentre sur les moyennes de deux objets seulement. D'un point de vue historique, c'est d'ailleurs sous cet aspect qu'apparaît pour la première fois le concept de moyenne. L'idée de proportion, chère aux mathématiciens grecs, précède de loin l'idée d'agrégation et de synthèse que développera beaucoup plus tard la statistique quantitative. Aussi, est-il tentant et naturel de considérer la moyenne comme une loi de composition interne, simple transcription moderne de la moyenne pythagoricienne.

La seconde partie envisage un prolongement à plus de deux objets. Si l'on cherche toujours à minimiser le nombre d'hypothèses nécessaires à sa construction, avec l'idée de conserver suffisamment de liberté pour opérer plus aisément dans le domaine qualitatif, on sera tout de même conduit à enrichir le modèle dans le but d'établir certaines propriétés locales. Ensuite, après avoir défini la moyenne pondérée, on examine les relations de correspondance entre moyennes pondérées et non pondérées. Et pour terminer, on énonce les propriétés barycentriques dont jouissent toutes les moyennes pondérées ou non.

À la manière des conditions de Yule [6], nous indiquons, ci-après, la liste des préceptes que doit satisfaire l'opérateur moyenne. Ainsi, nous exigeons de lui :

1. qu'il soit opérationnel dans le domaine quantitatif, comme dans le domaine qualitatif ;

2. qu'il contienne, comme cas particuliers, toutes les moyennes usuelles déjà existantes ;

3. qu'il soit d'exécution et d'interprétation aussi simple et immédiate que possible ;

4. qu'il tienne compte de toutes les données mises en jeu ;

5. qu'il délivre une et une seule réponse à chaque exécution ;

6. qu'il soit de même nature que les éléments mis en jeu ; mais non, nécessairement, l'un de ceux-là ;

7. qu'il évoque autant l'idée d'agrégation que celle de position intermédiaire ;

8. qu'il satisfasse les deux règles de bon sens suivantes :

- Règle de la valeur constante : la moyenne d'objets identiques est cet objet

- Règle de la valeur intermédiaire : toute moyenne se situe entre deux extrêmes. 


\section{MOYENNE DE DEUX OBJETS}

\subsection{Définition axiomatique}

Considérons un ensemble $\mathrm{E}$, muni d'une loi de composition interne, notée * , et d'une relation d'ordre, notée $\leq$, de sorte que l'ensemble ordonné $(\mathrm{E}, \leq)$ soit un treillis. La structure, notée $(\mathrm{E}, *, \leq)$, sur laquelle nous envisageons de construire une opération dite : moyenne selon la loi *, est donc un «magma» (ensemble doté d'une loi de composition interne) «réticulé» (existence d'une borne supérieure, notée Sup, et d'une borne inférieure, notée Inf, pour chaque paire d'éléments).

a) Moyenne de deux éléments, selon la loi $*$.

On appelle moyenne selon la loi $*$, toute application (lorsqu'elle existe), notée :

$$
\begin{aligned}
m: \mathrm{E} \square \mathrm{E} \square \mathrm{E} \\
(\mathrm{a}, \mathrm{b}) \mapsto m(\mathrm{a}, \mathrm{b})
\end{aligned}
$$

qui satisfait les deux axiomes suivants : pour tout élément $\mathrm{a}$ et $\mathrm{b}$ de $\mathrm{E}$,

Axiome $1: \operatorname{Inf}(\mathrm{a}, \mathrm{b}) \square m(\mathrm{a}, \mathrm{b}) \square \operatorname{Sup}(\mathrm{a}, \mathrm{b})$

Axiome 2 : L'élément $m(\mathrm{a}, \mathrm{b})$ est l'unique solution de l'équation : $\mathrm{x} * \mathrm{x} !=! \mathrm{a} * \mathrm{~b}$,

où $\mathrm{x}$, élément de $\mathrm{E}$, désigne l'inconnue de l'équation.

b) Remarques et commentaires

L'outillage mathématique requis ici est minimum. L'ensemble $\mathrm{E}$ est fini ou non, la relation d'ordre est totale ou partielle. La loi de composition interne est quelconque et n'est même pas supposée surjective.

Toutefois, lorsque deux structures sont combinées, ici la structure algébrique $(\mathrm{E}, *)$ et la structure d'ordre $(\mathrm{E}, \leq)$, il y a lieu de s'interroger sur leur compatibilité mutuelle. L'usage veut que, pour les structures familières (groupes, anneaux, etc.), la compatibilité soit reconnue de fait, et nous en tiendrons compte. Néanmoins, il est permis d'accepter «l'anticompatibilité» de ces deux relations sans que cela nuise, bien au contraire, au développement de la théorie ${ }^{2}$.

Ainsi, toute moyenne est, ici, assujettie à une structure donnée $(\mathrm{E}, *, \leq)$, et dérive donc d'une opération primaire, notée en la circonstance «*». C'est pourquoi, lorsqu'elle existe, nous parlerons de la moyenne selon la loi «*», et non d'une moyenne, étant entendu qu'une loi ne délivre qu'une seule moyenne (réciproque fausse, car une même moyenne peut provenir de plusieurs lois distinctes).

Nous dirons que la structure $(\mathrm{E}, *, \leq)$ est moyennable, si la moyenne selon la loi «*» de E existe.

L'objet $m(\mathrm{a}, \mathrm{b})$ est également appelé moyenne de «a» et de «b», confondant ainsi, opération et résultat.

\footnotetext{
2. L'ordre est dit compatible avec la loi (donc à droite et à gauche) si et seulement si : $\square(a, b, c) a \square b \square(a \square c \square b \square c$ et $c \square a \square c \square b)$ Elle est dite anticompatible si et seulement si : $\square(a, b, c) a \square b \square(a \square c \geq b \square c$ et $c \square a \geq c \square b)$, étant entendu que l'ordre peut n'être ni l'un, ni l'autre.
} 
Les objets $\operatorname{Inf}(a, b)$ et $\operatorname{Sup}(a, b)$ sont nécessairement deux éléments comparables de E!; si bien que si $m$ existe, on a nécessairement:

$$
\operatorname{Inf}(a, b) \leq m(\operatorname{Inf}(a, b), \operatorname{Sup}(a, b)) \leq \operatorname{Sup}(a, b) .
$$

L'équation : $\mathrm{x} * \mathrm{x}=\mathrm{a} * \mathrm{~b}$, que dorénavant nous appellerons équation caractéristique, peut admettre a priori une ou plusieurs solutions, (i.e. en nombre fini, ou infini, dénombrable ou non) ou bien encore n'admettre aucune solution ; cela dépend de la structure $(\mathrm{E}, *, \leq)$.

Lorsque l'équation caractéristique n'admet pas de solution pour au moins un couple $(\mathrm{a}, \mathrm{b})$, nous dirons qu'il n'existe pas de moyenne sur E selon la loi «*» ou que $(\mathrm{E}, *, \leq)$ n'est pas moyennable. Il en sera de même s'il existe plusieurs solutions. D'ailleurs, dans ces cas, la relation «m» perdrait son statut d'application au sens mathématique du terme. Si plusieurs solutions s'avèrent possibles, l'expérience montre que l'on peut souvent restreindre l'ensemble $\mathrm{E}$, de sorte que l'équation susmentionnée n'admette qu'une seule solution pour tout «a et $\mathrm{b} »$ de $\mathrm{E}$.

Il est évident que l'unicité de l'application moyenne selon la loi «*» résulte de l'unicité exigée de la solution de l'équation caractéristique.

La moyenne selon une loi est elle-même une loi de composition interne. De plus, elle possède la propriété d'idempotence, à savoir pour tout «a» de $E: m(a, a)=a$. En effet, $\mathrm{x} !=! \mathrm{a}$ est solution évidente (et donc unique) de l'équation : $\mathrm{x} * \mathrm{x} !=! \mathrm{a} * \mathrm{a}$.

On vérifie aisément que les huit conditions que nous avons exigées, sont satisfaites, notamment, le respect de la double idée d'agrégation et d'intermédiaire.

En particulier, toutes les moyennes numériques classiques vérifient bien les huit conditions et les deux axiomes supra ; par exemple!: la moyenne arithmétique est la moyenne selon l'addition des nombres réels, la moyenne géométrique est la moyenne selon la multiplication des nombres réels positifs.

\section{c) Quelques exemples}

\section{$1^{\text {er }}$ exemple}

De manière générale, on peut définir la moyenne d'ordre $r$ (où $r$ est un nombre réel), en considérant la loi de composition interne définie dans l'ensemble $\mathbb{R}$ des nombres réels (ou dans une de ses parties selon les cas d'existence) par :

$$
a * b !=! f(a)+f(b)
$$

où $\mathrm{f}(\mathrm{x}) !=! \mathrm{x}^{\mathrm{r}}$ si $\mathrm{r} ! \neq ! 0$,

et $\mathrm{f}(\mathrm{x}) !=! \ln (\mathrm{x})$ pour $\mathrm{r} !=! 0$ (prolongement par continuité).

L'équation caractéristique $: \mathrm{x} * \mathrm{x}=\mathrm{a} * \mathrm{~b}$ est équivalente à : $! 2 \mathrm{f}(\mathrm{x}) !=! \mathrm{f}(\mathrm{a})+\mathrm{f}(\mathrm{b})$, et

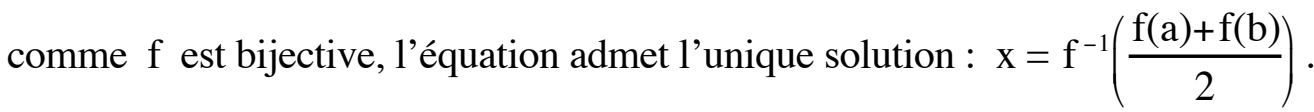

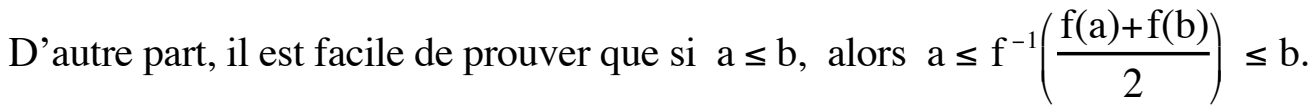

Ainsi, a-t-on plus précisément : 


$$
\begin{aligned}
& m_{\mathrm{r}}(\mathrm{a}, \mathrm{b})=\frac{\square \mathrm{a}^{\mathrm{r}}+\mathrm{b}^{\mathrm{r}} !^{\frac{1}{r}}}{2} \text { pour } \mathrm{r} ! \neq ! 0, \\
& m_{0}(\mathrm{a}, \mathrm{b})=\sqrt[2]{\mathrm{ab}} \quad \text { pour } \mathrm{r}=0 .
\end{aligned}
$$

En particulier : si $r=-1$, c'est la moyenne harmonique ; si $r=0$, c'est la moyenne géométrique; si $\mathrm{r}=1$, c'est la moyenne arithmétique; et si $\mathrm{r}=2$, c'est la moyenne quadratique.

En outre, lorsque $r$ tend vers l'infini (respectivement vers moins l'infini), il est aisé de prouver que la moyenne d'ordre $r$ tend vers la valeur maximale de $a$ et de $b$ (respectivement vers la valeur minimale); si bien que l'on peut poser : $m_{+}(\mathrm{a}, \mathrm{b}) !=! \operatorname{Sup}(\mathrm{a}, \mathrm{b}) \quad$ et $\quad m_{\square}(\mathrm{a}, \mathrm{b}) !=! \operatorname{Inf}(\mathrm{a}, \mathrm{b})$.

Sur $\mathbb{R}_{+}^{*}$ on démontre que si $r_{1} \square r_{2}$, alors $m_{\square} \square m_{r_{1}} \square m_{r_{2}} \square m_{+}$

$2^{\mathrm{e}}$ exemple

Considérons la structure $(P(\mathrm{E}), \square, \square)$ où $P(\mathrm{E})$ désigne l'ensemble des parties de E!; il est aisé de vérifier que $\square$ est moyenne selon $\square$.

$3^{\mathrm{e}}$ exemple

Soit a!=! $\square_{0}, \square_{1} \square_{2} \cdots \square_{i} \cdots$ la représentation d'un réel positif par un développement décimal illimité ( $\square_{0}$ est un entier et les $\square_{i}$ sont des chiffres de 0 à 9).

Rappelons qu'un nombre réel non décimal admet un unique développement décimal illimité, tandis qu'un décimal admet deux développements décimaux illimités, l'un dit propre : à partir d'un certain rang toutes ses décimales sont égales à zéro (ex.: $3,70250000 \ldots$ qui conformément à l'usage s'écrit aussi 3,7025) ; l'autre dit impropre : à partir du même rang toutes ses décimales sont égalent à neuf (ex.: 3,70249999...).

Cela étant, considérons l'ensemble $\mathbb{R}_{+}$des nombres réels positifs ou nuls et leur représentation par un développement décimal illimité ; pour les nombres décimaux on ne tiendra compte, ici, que de leur représentation propre (et non impropre).

Pour tout réel positif ou nul!: $\mathrm{a}=\square_{0}, \square_{1} \square_{2} \cdots \square_{i} \cdots$ et $\mathrm{b}=\square_{0}, \square_{1} \square_{2} \cdots \square_{i} \cdots$, nous définissons dans $\mathbb{R}_{+}$la relation notée $« \Pi »$ par : $a \Pi b$ si et seulement si, ou bien pour tout entier $i$, on a : $\square_{i}=\square_{i}$ (i.e. $\mathrm{a}=\mathrm{b}$ ), ou bien $\square_{j}$ divise $\square_{j}$ avec $\ll j »$ le plus petit entier tel que $\square_{j} \neq \square_{j}$. On vérifie facilement que la relation « $\rceil$ » est une relation d'ordre partiel.

Définissons maintenant la loi de composition «*» dans $\mathbb{R}_{+}$de la façon suivante!: $\mathrm{a} * \mathrm{a}=\mathrm{a}$ et si $\mathrm{a} \neq \mathrm{b}$, alors $\mathrm{a} * \mathrm{~b}=\mathrm{c}$ avec $\mathrm{c}=\square_{0}, \square_{1} \square_{2} \cdots \square_{j \square 1} \square_{j}$ où $\square_{j}=\operatorname{pgcd}\left(\square_{j}, \square_{j}\right)$ avec $« j »$ le plus petit entier tel que $\square_{j} \neq \square_{j}$ (ex. : 23,70418*23,7853333...=23,78). On remarque que cette loi est commutative et surjective, mais n'est ni associative ni régulière (i.e. il existe des éléments non réguliers).

Montrons, par contre, que cette loi est compatible avec l'ordre. Soit a $\Pi$ b ; on peut supposer $\mathrm{a}<\mathrm{b}$, car le cas $\mathrm{a}=\mathrm{b}$ est trivial. Donc, il existe un entier $j$ tel que : $\square_{0}=\square_{0}$, $\square_{1}=\square_{1}, \ldots, \square_{j \square 1}=\square_{j \square 1}$, et $\square_{j} \neq \square_{j}$ avec $\square_{j}$ divise $\square_{j}$. 
Soit $\mathrm{c}=\square_{0}, \square_{1} \square_{2} \cdots \square_{i} \cdots$. Si $\mathrm{a}=\mathrm{c}$, alors $\mathrm{a} * \mathrm{c}=\mathrm{a}=\square_{0}, \square_{1} \square_{2} \cdots \square_{i} \cdots$.

On a : $\mathrm{b} * \mathrm{c}=\mathrm{b} * \mathrm{a}=\square_{0}, \square_{1} \square_{2} \cdots \square_{j \square 1} \square_{j}$ car $\operatorname{pgcd}\left(\square_{j}, \square_{j}\right)=\square_{j}$ puisque $\square_{j}$ divise $\square_{j}$.

Soit, alors, un entier $k$ tel que $k>j$; de deux chose l'une, ou bien il existe $\square_{k} \neq 0$ et dans ce cas on a : $\mathrm{a} * \mathrm{c} \Pi \mathrm{b} * \mathrm{c}$ puisque $\square_{k}$ divise 0 ; ou bien pour tout $k>j$, on a $\square_{k}=0$ et dans ce cas $\mathrm{a} * \mathrm{c}=\mathrm{b} * \mathrm{c}$.

Supposons maintenant, $\mathrm{a} \neq \mathrm{c}$; et soit $k$ le plus petit entier tel que $\square_{k} \neq \square_{k}$. Selon la position de celui-ci, nous avons trois cas à examiner :

$1^{\text {er }} \quad$ cas! $: ! k<j . \quad$ On a!:!a*c! $=! \square_{0}, \square_{1} \square_{2} \cdots \square_{k} \quad$ avec $\square_{k}=\operatorname{pgcd}\left(\square_{k}, \square_{k}\right) \quad$ et $\mathrm{b} * \mathrm{c} !=! \square_{0}, \square_{1} \square_{2} \cdots \square_{k}$ avec $\square_{k}=\operatorname{pgcd}\left(\square_{k}, \square_{k}\right)$. Or, $\square_{k}=\square_{k} ;$ donc a*c=b*c.

$2^{\mathrm{e}} \quad$ cas $!: ! k=j . \quad$ On $\quad \mathrm{a} !: ! \mathrm{a} * \mathrm{c} !=! \square_{0}, \square_{1} \square_{2} \cdots \square_{j} \quad$ avec $\quad \square_{j}=\operatorname{pgcd}\left(\square_{j}, \square_{j}\right) \quad$ et $\mathrm{b} * \mathrm{c} !=! \square_{0}, \square_{1} \square_{2} \cdots \square_{j}$ avec $\square_{j}=\operatorname{pgcd}\left(\square_{j}, \square_{j}\right)$. Or, $\square_{j}$ divise $\square_{j} ;$ donc $\square_{j}$ divise $\square_{j}$ et par conséquent : $\mathrm{a} * \mathrm{c}<\mathrm{b} * \mathrm{c}$.

$3^{\mathrm{e}} \quad$ cas!:! $k>j$. On a!:!a*c! $=! \square_{0}, \square_{1} \square_{2} \cdots \square_{j} \cdots \square_{k} \quad$ avec $\square_{k}=\operatorname{pgcd}\left(\square_{k}, \square_{k}\right) \quad$ et $\mathrm{b} * \mathrm{c} !=! \square_{0}, \square_{1} \square_{2} \cdots \square_{j} \cdots \square$ avec $\square_{k}=\operatorname{pgcd}\left(\square_{k}, \square_{k}\right)$. Or, $\square_{j}$ divise $\square_{j} ;$ donc a*c! $<! \mathrm{b} * \mathrm{c}$.

Il nous reste à démontrer que $\left(\mathbb{R}_{+}, \Pi\right)$ est un treillis. Remarquons au passage, que zéro est l'élément maximum de $\mathbb{R}_{+}$(car tout entier non nul divise zéro) et que par conséquent : $\operatorname{Sup} \mathbb{R}_{+}=0$. De même, le nombre rationnel $10 / 9=1,111 \ldots$ est l'élément minimum de $\mathbb{R}_{+}$(car 1 divise tout entier) et que par conséquent : $\operatorname{Inf} \mathbb{R}_{+}=10 / 9$.

Cela étant, montrons que $\left(\mathbb{R}_{+}, \Pi\right)$ est un Inf-demi-treillis. Soient deux éléments a et $b$ non comparables (lorsqu'ils le sont, le plus grand minorant de cette paire est évidemment le plus petit des deux). Puisque $\mathrm{a} \neq \mathrm{b}$, il existe un entier $j$ pour lequel on a d'une part : pour tout $k<j, \square_{k}=\square_{k}$ et d'autre part : $\square_{j} \neq \square_{j} \neq 1$ avec $\operatorname{pgcd}\left(\square_{j}, \square_{j}\right)=1$. Ainsi, le nombre décimal $\square_{0}, \square_{1} \square_{2} \cdots \square_{j \square 1} 1$ est un minorant commun à a et à b. Or, tout minorant commun de a et de b est de la forme : $\square_{0}, \square_{1} \square_{2} \cdots \square_{j \square 1} 1 \square_{j+1} \cdots \square_{k} \cdots$ avec $\square_{k}$ un chiffre quelconque de 0 à 9 ; or tous ces nombres minorent $\square_{0}, \square_{1} \square_{2} \cdots \square_{j \square 1} 1$, puisque pour $\square_{k} \neq 0, \square_{k}$ divise 0 . Donc, $\square_{0}, \square_{1} \square_{2} \cdots \square_{j \square 1} 1$ est le plus grand des minorants communs de $a$ et $b$. Finalement, pour tout $a$ et $b$ non comparables, on $a$ : $\operatorname{Inf}(a, b)=a * b$ qui est un nombre décimal.

Montrons que $\left(\mathbb{R}_{+}, \Pi\right)$ est un Sup-demi-treillis. Soient deux éléments a et $b$ non comparables. Distinguons deux cas :

$1^{\text {er }}$ cas : $\square_{0} \neq \square_{0}$. Comme a et $b$ ne sont pas comparables, $\square_{0} \neq 0, \square_{0} \neq 0$ et $\operatorname{pgcd}\left(\square_{0}, \square_{0}\right)=1$. Posons $p_{0}=\operatorname{ppcm}\left(\square_{0}, \square_{0}\right)$; tout majorant commun de a et $\mathrm{b}$ est de la forme : $\left(\square_{0} \square p_{0}\right), \square_{1} \cdots \square_{i} \cdots$ où les $\left\langle\square_{i} »\right.$ sont des chiffres de 0 à 9 . Le plus petit d'entre eux existe, et vaut $: p_{0}, 111 \cdots 1 \cdots$. Donc $\operatorname{Sup}(\mathrm{a}, \mathrm{b})=p_{0}, 111 \cdots 1 \cdots$.

$2^{\mathrm{e}}$ cas : $\square_{0}=\square_{0}$. Puisque $\mathrm{a} \neq \mathrm{b}$, il existe un entier $j$ pour lequel on a d'une part : pour tout $k<j, \square_{k}=\square_{k}$ et d'autre part : $\square_{j} \neq \square_{j} \neq 1$ avec $\operatorname{pgcd}\left(\square_{j}, \square_{j}\right)=1$. Posons $p_{j}=\operatorname{ppcm}\left(\square_{j}, \square_{j}\right)$. Si $p_{j}<10$ (d'ailleurs dans ce cas, la seule valeur possible est $p_{j} !=$ 6), alors les majorants communs de a et de $b$ sont de la forme!: 
$\square_{0}, \square_{1} \square_{2} \cdots \square_{j \square 1} p_{j} \square_{j+1} \cdots \square_{k} \cdots \quad$ ou bien de la forme : $\square_{0}, \square_{1} \square_{2} \cdots \square_{j \square 1} 0 \square_{j+1} \cdots \square_{k} \cdots$ avec $\square_{k}$ un chiffre quelconque de 0 à 9 . Or, le plus petit d'entre eux existe et vaut: $\square_{0}, \square_{1} \square_{2} \cdots \square_{j \square 1} p_{j} 11 \cdots 1 \cdots$, car $p_{j}$ divise 0 , et 1 divise tout entier. Si $p_{j}>10$, alors il est clair que $\square_{0}, \square_{1} \square_{2} \cdots \square_{j \square 1} 111 \cdots 1 \cdots$ est le plus petit majorant commun de a et de $b$.

En résumé, la borne supérieure de deux éléments $\mathrm{a}$ et $\mathrm{b}$ non comparables est selon le cas : $\operatorname{Sup}(a, b)=\operatorname{ppcm}\left(\square_{0}, \square_{0}\right), 111 \cdots$ ou bien $\operatorname{Sup}(a, b)=\square_{0}, \square_{1} \square_{2} \cdots \square_{j \square 1} 611 \cdots 1 \cdots$ ou bien encore $\operatorname{Sup}(\mathrm{a}, \mathrm{b})=\square_{0}, \square_{1} \square_{2} \cdots \square_{j \square 1} 011 \cdots 1 \cdots$.

La structure $(\mathbb{R}, *, П)$ est donc bien un magma réticulé moyennable, de moyenne la loi de composition interne «*». On pourra remarquer que la moyenne de deux réels distincts est toujours un nombre décimal.

Consignons dans un tableau quelques résultats.

\begin{tabular}{ccccc}
\hline $\mathrm{a}$ & $\mathrm{b}$ & $\operatorname{Inf}(\mathrm{a}, \mathrm{b})$ & $\mathrm{m}(\mathrm{a}, \mathrm{b})$ & $\operatorname{Sup}(\mathrm{a}, \mathrm{b})$ \\
\hline 6 & 9 & 3 & 3 & $18,11 \ldots=163 / 9$ \\
4,27 & 4,268 & 4,21 & 4,21 & $4,2011 \ldots=3781 / 900$ \\
$9,0101 \ldots$ & $9,77 \ldots$ & $9,77 \ldots=88 / 9$ & 9,7 & $9,011 \ldots=811 / 90$ \\
0,0567 & $0,05959 \ldots$ & 0,053 & 0,053 & $0,05011 \ldots=451 / 9000$ \\
5,403 & $5,27878 \ldots$ & $5,27878 \ldots=5273 / 999$ & 5,2 & 5,403 \\
1,8036 & 1,80279 & 1,801 & 1,801 & $1,80611 \ldots=16255 / 9000$ \\
$\sqrt{2}$ & $\sqrt{3}$ & 1,1 & 1,1 & $1,011 \ldots=91 / 90$ \\
$\sqrt{18}$ & $\sqrt{19}$ & 4,1 & 4,1 & $4,611 \ldots=415 / 90$ \\
$\square$ & $\square$ & $\pi$ & $\square$ & $\pi$ \\
$\square$ & $22 / 7$ & $\pi$ & 3,141 & $3,1428571428 \ldots=22 / 7$ \\
\hline
\end{tabular}

\subsection{Propriétés algébriques}

On suppose, par la suite, la structure $(\mathrm{E}, *, \leq)$ moyennable.

On appelle «carré» l'application qui a tout «a» de E associe «a*a» de E.

a) Sans hypothèse supplémentaire.

PROPOSITION 2.1 : L'opération «carré» est une application injective

c'est-à-dire : $\square(\mathrm{a}, \mathrm{b}) \square \mathrm{E}^{2} \quad \mathrm{a} \square \mathrm{a}=\mathrm{b} \square \mathrm{b} \square \mathrm{a}=\mathrm{b}$

Preuve : En vertu de l'unicité de la solution de l'équation caractéristique.

PROPOSITION 2.2 : La moyenne est une application surjective

c'est-à-dire : $\square \mathrm{a} \square \mathrm{E} \square(\mathrm{b}, \mathrm{c}) \square \mathrm{E}^{2}, \quad \mathrm{a}=m(\mathrm{~b}, \mathrm{c})$

Preuve : Toute application idempotente est évidemment surjective. 
PROPOSITION 2.3 : La moyenne n'est pas une application injective (sauf si $|\mathrm{E}|=1$ ).

c'est-à-dire : $\square(\mathrm{a}, \mathrm{b}, \mathrm{c}, \mathrm{d}) \square \mathrm{E}^{4}, \quad m(\mathrm{a}, \mathrm{b})=m(\mathrm{c}, \mathrm{d})$ et $(\mathrm{a}, \mathrm{b}) \neq(\mathrm{c}, \mathrm{d})$

Preuve : Soit cardE $>1$; autrement dit, il existe au moins deux éléments «a» et «b» distincts. Raisonnons par l'absurde en supposant $m$ injective. Pour tout «a» différent de $\langle\mathrm{b} »$ on $\mathrm{a}: m(\mathrm{a}, \mathrm{b})=m(m(\mathrm{a}, \mathrm{b}), m(\mathrm{a}, \mathrm{b}))$, or $m$ étant injective il vient $:(\mathrm{a}, \mathrm{b})=$ $(m(\mathrm{a}, \mathrm{b}), m(\mathrm{a}, \mathrm{b}))$; et par égalité des couples : $\mathrm{a}=m(\mathrm{a}, \mathrm{b})$ et $\mathrm{b}=m(\mathrm{a}, \mathrm{b}) \quad \mathrm{c}$ 'est dire que $\mathrm{a}=$ $\mathrm{b}$, ce qui est contraire à l'hypothèse.

b) Commutativité

PROPOSITION 2.4 : La moyenne est commutative si et seulement si la loi * l'est.

c'est-à-dire : $\square(\mathrm{a}, \mathrm{b}) \square \mathrm{E}^{2}, \quad m(\mathrm{a}, \mathrm{b})=m(\mathrm{~b}, \mathrm{a})$

Preuves : ( $\square$ ) On suppose $m$ commutative. Par définition de $m$, on a : $\mathrm{a} \square \mathrm{b}=m(\mathrm{a}, \mathrm{b}) \square m(\mathrm{a}, \mathrm{b})$ et par hypothèse $m(\mathrm{a}, \mathrm{b}) \square m(\mathrm{a}, \mathrm{b})=m(\mathrm{~b}, \mathrm{a}) \square m(\mathrm{~b}, \mathrm{a}) \quad \mathrm{d}$ 'où $\mathrm{a} \square \mathrm{b}=\mathrm{b} \square \mathrm{a}$.

( $\square$ ) On suppose * commutative Par définition de $m$, on a : $m(\mathrm{a}, \mathrm{b}) \square m(\mathrm{a}, \mathrm{b})=\mathrm{a} \square \mathrm{b}$ et par hypothèse $m(\mathrm{a}, \mathrm{b}) \square m(\mathrm{a}, \mathrm{b})=\mathrm{a} \square \mathrm{b}=\mathrm{b} \square \mathrm{a}=m(\mathrm{~b}, \mathrm{a}) \square m(\mathrm{~b}, \mathrm{a})$ d'où d'après la Proposition $2.1(\S 2.2-\mathrm{a})$ on $\mathrm{a}: m(\mathrm{a}, \mathrm{b})=m(\mathrm{~b}, \mathrm{a})$.

c) Régularité

PROPOSITION 2.5 : Un élément «c» de E est régulier à droite (respectivement à gauche) pour $m$, si et seulement si, il l'est à droite (respectivement à gauche) pour la loil*.

c'est-à-dire : $\square(\mathrm{a}, \mathrm{b}) \square \mathrm{E}^{2}, \quad m(\mathrm{a}, \mathrm{c})=m(\mathrm{~b}, \mathrm{c}) \square \mathrm{a}=\mathrm{b}$

Preuve : On a par définition de $m: \mathrm{a} \square \mathrm{c}=\mathrm{b} \square \mathrm{c} \square m(\mathrm{a}, \mathrm{c}) \square m(\mathrm{a}, \mathrm{c})=m(\mathrm{~b}, \mathrm{c}) \square m(\mathrm{~b}, \mathrm{c})$, et d'après la proposition $2.1(\S 2.2-\mathrm{a})$ on $\mathrm{a}: m(\mathrm{a}, \mathrm{c})=m(\mathrm{~b}, \mathrm{c})$,

donc «c» est régulier pour $m$ ssi il l'est pour $*$, car dans les deux cas $\mathrm{a}=\mathrm{b}$.

PROPOSITION 2.6 : Si la loi * est régulière (à droite et à gauche), alors la moyenne $m$ selon cette loi, n'est pas une loi associative.

c'est-à-dire : $\square(\mathrm{a}, \mathrm{b}, \mathrm{c}) \square \mathrm{E}^{3}, \quad m(\mathrm{a}, m(\mathrm{~b}, \mathrm{c})) \neq m(m(\mathrm{a}, \mathrm{b}), \mathrm{c})$

Preuve: (Étant entendu qu'une loi de composition définie sur $\mathrm{E}$ est dite régulière, lorsque tout élément de $\mathrm{E}$ est régulier à gauche et à droite.)

Démontrons par l'absurde en supposant $m$ associative. Pour tout $\mathrm{a}, \mathrm{b}$ et $\mathrm{c}$ de $\mathrm{E}$ on aurait : $m(\mathrm{a}, m(\mathrm{~b}, \mathrm{c}))=m(m(\mathrm{a}, \mathrm{b}), \mathrm{c})$; en particulier pour $\mathrm{a}=\mathrm{b}$ et $\mathrm{c} \neq \mathrm{b}$ il viendrait : $m(\mathrm{a}, m(\mathrm{a}, \mathrm{c}))=m(a, \mathrm{c})$. D'où $\quad \mathrm{a} \square m(\mathrm{a}, \mathrm{c})=a \square \mathrm{c}$ et par régularité à gauche, $m(\mathrm{a}, \mathrm{c})=\mathrm{c}$. De même on en déduirait que $\mathrm{a} \square \mathrm{c}=\mathrm{c} \square \mathrm{c}$ et par régularité à droite, $\mathrm{a}=\mathrm{c}$; ce qui est contraire à l'hypothèse.

d) Élément absorbant 
PROPOSITION 2.7 : Un élément «0» de E est absorbant à droite (respectivement à gauche) pour la loi * si, il l'est à droite (respectivement à gauche) pour $m$.

c'est-à-dire : $\square \mathrm{a} \square \mathrm{E}, \quad m(\mathrm{a}, \mathrm{o})=\mathrm{o}$

Preuve : Soit «o» un élément de $\mathrm{E}$ absorbant pour *, on a pour tout «a» de E : $m(\mathrm{a}, \mathrm{o}) \square m(\mathrm{a}, \mathrm{o})=\mathrm{a} \square \mathrm{o}=\mathrm{o}=\mathrm{o} \square \mathrm{o}$; et d'après la proposition 2.1. (§ 2.2-a) on a!: $m(\mathrm{a}, \mathrm{o}) !=$ o.

La réciproque est fausse, car si «o» est un élément de $\mathrm{E}$ absorbant pour $m$, on a pour tout «a»de $\mathrm{E}: \mathrm{a} \square \mathrm{o}=m(\mathrm{a}, \mathrm{o}) \square m(\mathrm{a}, \mathrm{o})=\mathrm{o} \square \mathrm{o}$; mais rien ne prouve que $\mathrm{o} \square \mathrm{o}=\mathrm{o}$.

e) Cas d'une loi $*$, à la fois associative et commutative

PROPOSITION 2.8 : La loi * est distributive par rapport à la loi moyenne $m$,

c'est-à-dire : $\square \mathrm{h} \square \mathrm{E}, \square(\mathrm{a}, \mathrm{b}) \square \mathrm{E}^{2} \quad m(\mathrm{~h} \square \mathrm{a}, \mathrm{h} \square \mathrm{b})=\mathrm{h} \square m(\mathrm{a}, \mathrm{b})$.

Preuve : On a par définition : $m(\mathrm{~h} \square \mathrm{a}, \mathrm{h} \square \mathrm{b}) \square m(\mathrm{~h} \square \mathrm{a}, \mathrm{h} \square \mathrm{b})=(\mathrm{h} \square \mathrm{a}) \square(\mathrm{h} \square \mathrm{b})$ et par associativité et commutativité $m(\mathrm{~h} \square \mathrm{a}, \mathrm{h} \square \mathrm{b}) \square m(\mathrm{~h} \square \mathrm{a}, \mathrm{h} \square \mathrm{b})=\mathrm{h} \square \mathrm{h} \square \mathrm{a} \square \mathrm{b}$; de même $\mathrm{h} \square m(\mathrm{a}, \mathrm{b}) \square \mathrm{h} \square m(\mathrm{a}, \mathrm{b})=\mathrm{h} \square \mathrm{h} \square m(\mathrm{a}, \mathrm{b}) \square m(\mathrm{a}, \mathrm{b})=\mathrm{h} \square \mathrm{h} \square \mathrm{a} \square \mathrm{b}$ !; !donc d'après la proposition $2.1(\S 2.2-\mathrm{a})$ on $\mathrm{a}: m(\mathrm{~h} \square \mathrm{a}, \mathrm{h} \square \mathrm{b})=\mathrm{h} \square m(\mathrm{a}, \mathrm{b})$

PROPOSITION 2.9 : règle dite «échange des moyens»

Pour tout élément $\mathrm{a}_{\mathrm{i}}$ et $\mathrm{b}_{\mathrm{i}}$ de $\mathrm{E}$, on $\mathrm{a}$ :

$$
m\left(\mathrm{a}_{1} \square \cdots \square \mathrm{a}_{n}, \mathrm{~b}_{1} \square \cdots \square \mathrm{b}_{n}\right)=m\left(\mathrm{a}_{1}, \mathrm{~b}_{1}\right) \square \cdots \square m\left(\mathrm{a}_{\mathrm{n}}, \mathrm{b}_{\mathrm{n}}\right)
$$

En d'autres termes, l'application $m$ est un homomorphisme de $\left(\mathrm{E}^{\mathrm{n}} \square \mathrm{E}^{\mathrm{n}}, \bullet\right)$ dans $(\mathrm{E}, \square)$ où «•» est la loi produit de $\mathrm{E}^{\mathrm{n}} \quad(\mathrm{n}$ entier non nul) définie par!: $\left(a_{1}, \cdots, a_{n}\right) \bullet\left(b_{1}, \cdots, b_{n}\right)=\left(a_{1} \square b_{1}, \cdots, a_{n} \square b_{n}\right)$.

Preuve : similaire à la proposition 2.8 (§2.2-e).

Exemple : Considérons, dans l'ensemble des nombres rationnels strictement positifs $\mathbb{Q}_{+}^{*}$, la loi $*$ définie par: pour tout a et $b$ de $\mathbb{Q}_{+}^{*} a \square b=\frac{a b}{a+b}$.

On vérifie aisément que cette loi est commutative, associative, régulière, surjective, non idempotente et compatible avec la relation d'ordre total usuel $\Pi$ de $\mathbb{Q}_{+}^{*}$. En outre, il existe une unique solution de l'équation caractéristique : $x \square x=\mathrm{a} \square \mathrm{b}$, à savoir : $x=\frac{2 \mathrm{ab}}{\mathrm{a}+\mathrm{b}}$.

On montre facilement que si $a b$ alors $a \frac{2 a b}{a+b} \square b$; si bien que la moyenne existe et vaut : $m(\mathrm{a}, \mathrm{b})=\frac{2 \mathrm{ab}}{\mathrm{a}+\mathrm{b}}$.

Vérifions, sur cet exemple, les propriétés 8 et 9. 


$$
m(h \square \mathrm{a}, h \square \mathrm{b})=\frac{2(h \square \mathrm{a})(h \square \mathrm{b})}{(h \square \mathrm{a})+(h \square \mathrm{b})}=\frac{2 \mathrm{ab} h}{2 \mathrm{ab}+h(\mathrm{a}+\mathrm{b})} ; \text { or } h \square m(\mathrm{a}, \mathrm{b})=\frac{2 \mathrm{ab} h}{2 \mathrm{ab}+h(\mathrm{a}+\mathrm{b})}
$$

De même calculons : $m(\mathrm{a} \square \mathrm{b}, \mathrm{c} \square \mathrm{d})=\frac{2(\mathrm{a} \square \mathrm{b})(\mathrm{c} \square \mathrm{d})}{(\mathrm{a} \square \mathrm{b})+(\mathrm{c} \square \mathrm{d})}=\frac{2 \mathrm{abcd}}{\mathrm{abc}+\mathrm{abd}+\mathrm{acd}+\mathrm{bcd}}$ or, $m(\mathrm{a}, \mathrm{c}) \square m(\mathrm{~b}, \mathrm{~d})=\frac{2 \mathrm{ac}}{\mathrm{a}+\mathrm{c}} \square \frac{2 \mathrm{bd}}{\mathrm{b}+\mathrm{d}}=\frac{2 \mathrm{abcd}}{\mathrm{abc}+\mathrm{abd}+\mathrm{acd}+\mathrm{bcd}}$.

Remarquons que la loi définie par : $a \square b=\frac{a+b}{a b}$ procure la même moyenne, mais bien qu'elle soit aussi commutative, surjective, non idempotente et régulière, elle n'est ni associative ni compatible (mais anti-compatible) avec la relation d'ordre.

f) La structure $(\mathrm{E}, *, \leq)$ est un groupe réticulé

On suppose que $(\mathrm{E}, *)$ possède une structure de groupe.

On note «e» son élément neutre et «a'» le symétrique de «a».

$\mathrm{Si}(\mathrm{E}, *, \leq)$ est moyennable, on dit que $(\mathrm{E}, *, \leq)$ est un groupe moyennable.

On définit une «multiplication» externe dans (E,*) dont le domaine opérateur est l'ensemble des entiers relatifs $\mathbb{Z}$, par :

$\cdot \mathbb{Z} \sqcap \mathrm{E} \sqcap \mathrm{E}$

$$
\begin{aligned}
(p, \mathrm{a}) \mapsto p \cdot \mathrm{a} \quad & \text { si } p>0 \text { alors } p \cdot \mathrm{a}=\underbrace{\mathrm{a} \square \mathrm{a} \square \cdots \square \mathrm{a}}_{p \text { fois }} \\
& \text { si } p=0 \text { alors } p \cdot \mathrm{a}=\mathrm{e} \\
& \text { si } p<0 \text { alors } p \cdot \mathrm{a}=(\square p) \cdot \mathrm{a}[
\end{aligned}
$$

PROPOSITION 2.10 : Dans un groupe moyennable $(\mathrm{E}, *, \leq)$, on a :

$$
\square(\mathrm{a}, \mathrm{b}) \square \mathrm{E}^{2} \quad \mathrm{~m}(\mathrm{a}, \mathrm{b})=\mathrm{e} \square \mathrm{a}=\mathrm{b},
$$

Preuves: ( $\square$ ) Par définition on $\mathrm{a}$ !: $m(\mathrm{a}, \mathrm{b}) \square m(\mathrm{a}, \mathrm{b})=\mathrm{a} \square \mathrm{b}$, par hypothèse $m(\mathrm{a}, \mathrm{b}) \square m(\mathrm{a}, \mathrm{b})=\mathrm{e} \square \mathrm{e}=\mathrm{e}$ donc $\mathrm{a} \square \mathrm{b}=\mathrm{e}$ et $\mathrm{a}=\mathrm{b}[$.

( $\square$ ) Soit $\mathrm{a}=\mathrm{b}[$, on a $m(\mathrm{~b} \square \mathrm{b}) \square m(\mathrm{~b} \square \mathrm{b})=\mathrm{b} \square \mathrm{b}=\mathrm{e}=\mathrm{e} \square \mathrm{e}$ donc d'après la proposition $2.1(\S 2.2-\mathrm{a}), m(\mathrm{~b} \square \mathrm{b})=\mathrm{e}$, c'est-à-dire $m(\mathrm{a}, \mathrm{b})=\mathrm{e}$.

PROPOSITION 2.11 : Dans un groupe moyennable $(\mathrm{E}, *, \leq)$, on a :

$$
\square(\mathrm{a}, \mathrm{b}) \square \mathrm{E}^{2} \quad[\mathrm{~m}(\mathrm{a}, \mathrm{b})]^{\prime}=\mathrm{m}\left(\mathrm{b}^{\prime}, \mathrm{a}^{\prime}\right)
$$

Preuve : On a : $m\left(\mathrm{~b}^{\prime}, \mathrm{a}^{\prime}\right) \square m\left(\mathrm{~b}^{\prime}, \mathrm{a}^{\prime}\right)=\mathrm{b} \square \mathrm{a}^{\prime}=(\mathrm{a} \square \mathrm{b})^{\prime}=[m(\mathrm{a}, \mathrm{b})]^{\prime}[m(\mathrm{a}, \mathrm{b})]^{\prime}$ d'où le résultat d'après la proposition $2.1(\S 2.2-\mathrm{a})$.

PROPOSITION 2.12 : Dans un groupe moyennable $(\mathrm{E}, *, \leq)$, l'équation d'inconnue $\mathrm{x}$ : $m(\mathrm{a}, \mathrm{x})=\mathrm{b}$, admet une unique solution, à savoir : $\mathrm{x}=\mathrm{a} \square \mathrm{b} \square \mathrm{b}$. 
Preuve : On a par définition : $m(\mathrm{a}, \mathrm{x}) \square m(\mathrm{a}, \mathrm{x})=\mathrm{a} \square \mathrm{x}$ et par hypothèse $m(\mathrm{a}, \mathrm{x}) \square m(\mathrm{a}, \mathrm{x})=\mathrm{b} \square \mathrm{b}$, d'où $\mathrm{a} \square \mathrm{x}=\mathrm{b} \square \mathrm{b}$. Or $\mathrm{a} \square \mathrm{a} \square \mathrm{x}=\mathrm{a} \square \mathrm{b} \square \mathrm{b}$, donc $\mathrm{x}=\mathrm{a} \square \mathrm{b} \square \mathrm{b}$.

PROPOSITION 2.13 : Dans un groupe moyennable $(\mathrm{E}, *, \leq)$ abélien, on a :

$$
\square \mathrm{p} \square \mathbb{Z}, \square(\mathrm{a}, \mathrm{b}) \square \mathrm{E}^{2} \quad m(\mathrm{p} \cdot \mathrm{a}, \mathrm{p} \cdot \mathrm{b})=\mathrm{p} \cdot m(\mathrm{a}, \mathrm{b})
$$

Preuve : Puisque la loi est commutative et associative, on peut utiliser la proposition 2.9 ( $\S$ 2.2-e) dans le cas particulier où pour tout $i$ on $\mathrm{a}: \mathrm{a}_{\mathrm{i}}=\mathrm{a}$ et $\mathrm{b}_{\mathrm{i}}=\mathrm{b}$.

PROPOSITION 2.14 : Dans un groupe moyennable $(\mathrm{E}, *, \leq)$ abélien, on a :

$$
\square(\mathrm{a}, \mathrm{b}) \square \mathrm{E}^{2} \quad m\left[\mathrm{a} \square m\left(\mathrm{~b}^{\prime}, \mathrm{a}\right), \mathrm{b} \square m\left(\mathrm{~b}^{\prime}, \mathrm{a}\right)\right]=\mathrm{e} .
$$

Preuve $: ! 2 \cdot m\left[\mathrm{a} \square m\left(\mathrm{~b}^{\prime}, \mathrm{a}^{\prime}\right), \mathrm{b} \square m\left(\mathrm{~b}^{\prime}, \mathrm{a}^{\prime}\right)\right]=2 \cdot\left(\mathrm{a} \square \mathrm{b} \square\left(2 \cdot m\left(\mathrm{~b}^{\prime}, \mathrm{a}^{\prime}\right)\right)\right) \quad$ et $\quad \mathrm{d}$ 'aprè s $\quad 1 \mathrm{a}$ proposition 2.1 (§ 2.2-a) $m\left\lfloor\mathrm{a} \square m\left(\mathrm{~b}^{\prime}, \mathrm{a}^{\prime}\right), \mathrm{b} \square m\left(\mathrm{~b}^{\prime}, \mathrm{a}^{\prime}\right)\right\rfloor=\left(\mathrm{a} \square \mathrm{b} \square\left(2 \cdot m\left(\mathrm{~b}^{\prime}, \mathrm{a}^{\prime}\right)\right)\right)$; par suite $m\left\lfloor\mathrm{a} \square m\left(\mathrm{~b}^{\prime}, \mathrm{a}^{\prime}\right), \mathrm{b} \square m\left(\mathrm{~b}^{\prime}, \mathrm{a}^{\prime}\right)\right\rfloor=\mathrm{a} \square \mathrm{b} \square \mathrm{b} \mathrm{h}^{\prime}$ et donc $m\left\lfloor\mathrm{a} \square m\left(\mathrm{~b}^{\prime}, \mathrm{a}^{\prime}\right), \mathrm{b} \square m\left(\mathrm{~b}^{\prime}, \mathrm{a}^{\prime}\right)\right\rfloor=\mathrm{e}$.

\subsection{Structures moyennables}

a) Remarque préliminaire

Soit une structure $(\mathrm{E}, *, \leq)$ moyennable, de moyenne $m$.

On peut supposer (ce que nous ferons par la suite) que la loi $*$ est surjective, car si elle ne l'était pas, il est toujours possible de trouver une structure $(E, \quad \leq)$ de même moyenne $m$, avec pour loi , une loi surjective.

PROPOSITION 3.1 : Soit une structure $(\mathrm{E}, *, \leq)$ moyennable de moyenne $m$. selon la loi $*$. Il existe une loi surjective, de sorte que la structure $(\mathrm{E}, \quad \leq)$ soit moyennable, de moyenne selon la loi égale à la moyenne selon la loi $*$.

Preuve : Soient $m$ la moyenne selon la loi $*$ et $\mathrm{g}$ une bijection de E. Considérons la loi définie dans $\mathrm{E}$ par : $\mathrm{a} \quad \mathrm{b}=\mathrm{g}(m(\mathrm{a}, \mathrm{b}))$. Cette loi est surjective comme composée de l'application surjective $m$ et de la bijection $\mathrm{g}$. L'équation caractéristique : $\mathrm{x} \quad \mathrm{x}=\mathrm{a} \quad \mathrm{b}$ devient $g(\mathrm{x})=\mathrm{a} \quad \mathrm{b}$ en tenant compte de ce que $\mathrm{x} \quad \mathrm{x}=\mathrm{g}(m(\mathrm{x}, \mathrm{x}))=\mathrm{g}(\mathrm{x})$ !; d'où $\mathrm{x}=\mathrm{g}^{-1}(\mathrm{a} \quad \mathrm{b})=\mathrm{g}^{-1} \circ \mathrm{g}(m(\mathrm{a}, \mathrm{b}))=m(\mathrm{a}, \mathrm{b})$.

b) Lois idemfotentes

Pour la commodité de l'exposé, nous introduisons le néologisme ${ }^{3}$ «loi idemfotente» qui peut-être considéré comme une généralisation de la notion de loi idempotente (littéralement!: de même potentiel).

DÉFINITION : Soient, un magma réticulé $(\mathrm{E}, *, \leq)$ et la fonction $\mathrm{f}$ définie sur $\mathrm{E}$ par : pour tout $\mathrm{x}$ élément de $\mathrm{E}, \mathrm{f}(\mathrm{x})=\mathrm{x} \square \mathrm{x}$. Nous dirons que la loi $*$ est idemfotente, lorsque la fonction $\mathrm{f}$, associée à $*$, est bijective.

Nous dirons aussi parfois que la loi $*$ est f-idemfotente, pour plus de précision.

\footnotetext{
${ }^{3}$ Néologisme sans fondement étymologique, mais obtenu par contraction des deux termes : idempotent et fonction.
} 
Remarquons qu'une loi f-idemfotente est idempotente lorsque $\mathrm{f}=i d_{\mathrm{E}}$.

\section{PROPOSITION 3.2}

Si les relations, $*$ et $\leq$ sont compatibles, alors $\mathrm{f}$ est croissante.

Si les relations, $*$ et $\leq$ sont anticompatibles, alors $\mathrm{f}$ est décroissante.

Preuve : Pour l'exemple donnons celle où les relations, $*$ et $\leq$ sont supposées anticompatibles. Soient a et $\mathrm{b}$ deux éléments de $(\mathrm{E}, *, \leq)$ tels que : a $\square \mathrm{b}$. En composant par a à droite on obtient $\mathrm{b} \square \mathrm{a} \square \mathrm{a} \square \mathrm{a}$ et en composant par b à gauche on obtient $\mathrm{b} \square \mathrm{b} \square \mathrm{b} \square \mathrm{a}$; si bien que par transitivité on $\mathrm{a}: \mathrm{b} \square \mathrm{b} \square \mathrm{a} \square \mathrm{a}$.

PROPOSITION 3.3 : Si la loi $*$ est commutative et associative, alors l'application $\mathrm{f}$ est un endomorphisme, et un isomorphisme si de plus $*$ est f-idemfotente ;

c'est-à-dire : $\square(\mathrm{a}, \mathrm{b}) \square \mathrm{E}^{2}, \quad \mathrm{f}(\mathrm{a} \square \mathrm{b})=\mathrm{f}(\mathrm{a}) \square \mathrm{f}(\mathrm{b})$.

Preuve : endomorphisme de $(\mathrm{E}, *)$ évident.

DÉFINITION : Nous dirons qu'un magma réticulé $(\mathrm{E}, *, \leq)$ est une structure compatible (respectivement anti-compatible), lorsque la loi $*$ est compatible (respectivement anticompatible) avec la relation d'ordre $\square$.

DÉFINITION : Soit $(\mathrm{E}, \leq)$ un ensemble ordonné et $\mathrm{f}$ une application de $\mathrm{E}$ dans $\mathrm{E}$.

Nous dirons que $\mathrm{f}$ est un isomorphisme d'ordre si, $\mathrm{f}$ est bijective et si $\mathrm{f}$ et $\mathrm{f}^{-1}$ sont simultanément des bijections croissantes.

Nous dirons que $\mathrm{f}$ est un anti-isomorphisme d'ordre si, $\mathrm{f}$ est bijective et si $\mathrm{f}_{\text {et }} \mathrm{f}^{-1}$ sont simultanément des bijections décroissantes.

PROPOSITION 3.4 : Considérons une structure $(\mathrm{E}, *, \leq)$ compatible (respectivement anti-compatible), avec $\leq$ un ordre total.

Si la loi * est f-idemfotente, alors l'application $\mathrm{f}$ est un isomorphisme d'ordre (respectivement anti-isomorphisme d'ordre).

Preuve: (pour * compatible avec $\square$ totale). Posons $y_{1}=\mathrm{f}\left(x_{1}\right)$ et $y_{2}=\mathrm{f}\left(x_{2}\right)$. Si $y_{1} \square y_{2}$ montrons que $x_{1} \square x_{2}$. Or, puisque l'ordre est total, on a ou bien $x_{1} \square x_{2}$ ou bien $x_{1}>x_{2}$. Si c'était $x_{1}>x_{2}$, comme $\mathrm{f}$ est croissante on aurait $\mathrm{f}\left(x_{1}\right)>\mathrm{f}\left(x_{2}\right)$ c'està-dire $y_{1}>y_{2}$ ce qui n'est pas, par hypothèse. Donc $x_{1} \square x_{2}$, ce qui signifie $\mathrm{f}^{\square 1}$ croissante.

PROPOSITION 3.5 : Considérons une structure $(\mathrm{E}, *, \leq)$ compatible (respectivement anti-compatible), avec $\mathrm{E}$ un ensemble fini.

Si la loi * est f-idemfotente, alors l'application $\mathrm{f}$ est un isomorphisme d'ordre (respectivement anti-isomorphisme d'ordre). 
Preuves $: 1^{\mathrm{er}}$ cas. $*$ compatible avec $\square$. La bijection $\mathrm{f}$ est donc croissante, montrons que $\mathrm{f}^{-1}$ l'est aussi. Soit card(E) $=\mathrm{n}$; il existe donc (n!) bijections de E. Considérons pour $p$ entier l'application $\mathrm{f}^{\mathrm{p}}=\mathrm{f} \circ \mathrm{f} \circ \cdots \circ \mathrm{f}$ ( $\mathrm{p}$ fois), qui est aussi une bijection croissante par composition. Maintenant, considérons $p>(n !)$, alors il existe un entier $\mathrm{r}$ avec $\mathrm{r} \square \mathrm{n}$ !, tel que $\mathrm{f}^{\mathrm{p}}=\mathrm{f}^{\mathrm{r}}$ puisqu'il n'existe qu'un nombre fini (n!) de bijections. D'où $\mathrm{f}^{\mathrm{p} \square \mathrm{r}}=\mathrm{id}_{\mathrm{E}}$, c'est-à-dire : $\mathrm{f}^{\mathrm{p} \square \mathrm{r} \square 1}=\mathrm{f}^{\square 1}$ qui est croissante puisque $\mathrm{f}^{\mathrm{p} \square \mathrm{r} \square 1}$ est la composée de bijections croissantes.

$2^{\mathrm{e}}$ cas : * anticompatible avec $\square$. La bijection $\mathrm{f}$ est donc décroissante, montrons que $\mathrm{f}^{-1}$ l'est aussi. Pour $\mathrm{p}>(\mathrm{n} !)$, on a d'une part : $\mathrm{f}^{2 \mathrm{p}+1}$ est une fonction décroissante et d'autre part : il existe un entier $r$, tel que $\mathrm{f}^{2 \mathrm{p}+1}=\mathrm{f}^{\mathrm{r}}$ avec $《 \mathrm{r} »$ nécessairement impair, car sinon $\mathrm{f}^{r}$ serait croissante par composition. Par conséquent $\mathrm{f}^{2 \mathrm{p} \square \mathrm{r}}=\mathrm{f}^{\square 1}$; or $2 \mathrm{p}-\mathrm{r}$ est impair puisque $r$ aussi, donc $\mathrm{f}^{\square 1}$ est décroissante.

c) Conditions nécessaires et suffisantes pour que $(\mathrm{E}, *, \leq)$ soit moyennable.

\section{THÉORÈME 3.1 : Condition nécessaire}

Si le magma réticulé $(\mathrm{E}, *, \leq)$ est moyennable, alors la loi * est idemfotente.

Preuve : Pour tout «y» de E, il existe «a et $\mathrm{b} »$ de $\mathrm{E}$ tel que $\mathrm{y}=\mathrm{a} * \mathrm{~b}$ (car $*$ est supposée surjective). Mais comme $(\mathrm{E}, *, П)$ est moyennable, il existe une application de ExE dans $\mathrm{E}$, notée $m$, dont l'image $m(\mathrm{a}, \mathrm{b})$ de $(\mathrm{a}, \mathrm{b})$ est l'unique solution de l'équation $: \mathrm{f}(\mathrm{x})=\mathrm{x} * \mathrm{x}=\mathrm{a} * \mathrm{~b}$. Donc $\ll \mathrm{f} »$ est bijective et $m(\mathrm{a}, \mathrm{b})=\mathrm{f}^{\square 1}(\mathrm{a} \square \mathrm{b})$.

THÉORÈME de caractérisation : Condition nécessaire et suffisante

Un magma réticulé $(\mathrm{E}, *, \leq)$ est moyennable si et seulement si : il existe une bijection $\mathrm{f}$ de $\mathrm{E}$ dans $\mathrm{E}$, telle que pour tout $\mathrm{a}$ et $\mathrm{b}$ de $\mathrm{E}$ on ait :

$$
f^{\square 1}(a \square b) \square[\operatorname{Inf}(a, b), \operatorname{Sup}(a, b)]=\{x \square E, \operatorname{Inf}(a, b) \square x \square \operatorname{Sup}(a, b)\} .
$$

Preuve: (ப) Si $(\mathrm{E}, *, \leq)$ est moyennable, d'après le théorème $1(\S 2.3-\mathrm{c})$ la loi * est f-idemfotente et $m(\mathrm{a}, \mathrm{b})=\mathrm{f}^{\square 1}(\mathrm{a} \square \mathrm{b})$ donc le résultat est avéré.

( $\square$ ) Soit $\mathrm{f}$ une bijection de $\mathrm{E}$ telle que pour tout a et $\mathrm{b}$ de $\mathrm{E}$, on ait : $\operatorname{Inf}(a, b) \square f^{\square 1}(a \square b) \square \operatorname{Sup}(a, b)$. On a donc en particulier pour tout $x$ de $E$ : $\operatorname{Inf}(x, x) \square \mathrm{f}^{\square 1}(\mathrm{x} \square \mathrm{x}) \square \operatorname{Sup}(\mathrm{x}, \mathrm{x})$, c'est-à-dire $\mathrm{f}^{\square 1}(\mathrm{x} \square \mathrm{x})=\mathrm{x}$, ou encore $\mathrm{x} \square \mathrm{x}=\mathrm{f}(\mathrm{x})$. Comme $\mathrm{f}$ est bijective, l'équation caractéristique $\mathrm{x} \square \mathrm{x}=\mathrm{a} \square \mathrm{b}$ n'admet qu'une seule solution qui est : $x=f^{\square 1}(a \square b)$. En outre $f^{\square 1}(a \square b) \square[\operatorname{Inf}(a, b), \operatorname{Sup}(a, b)]$, donc $(E, *, \leq)$ est moyennable de moyenne $m(\mathrm{a}, \mathrm{b})=\mathrm{f}^{\square 1}(\mathrm{a} \square \mathrm{b})$.

THÉORÈME 3.2 : Condition suffisante

Soit un magma réticulé $(\mathrm{E}, *, \leq)$ compatible (respectivement anti-compatible). 
Si la loi * est f-idemfotente, avec $\mathrm{f}$ un isomorphisme d'ordre (respectivement un antiisomorphisme d'ordre), alors $(\mathrm{E}, *, \leq)$ est moyennable et sa moyenne $m$ est croissante, c'est-à-dire!: si $\mathrm{a} \square \mathrm{b}$ alors $m(\mathrm{a}, \mathrm{c}) \square m(\mathrm{~b}, \mathrm{c})$ pour tout $\mathrm{a}, \mathrm{b}$ et $\mathrm{c}$ de $\mathrm{E}$ (respectivement décroissante).

Preuve : Puisque $\mathrm{f}$ est bijective de $\mathrm{E}$ dans $\mathrm{E}$, pour tout «y» de E l'équation : $\mathrm{f}(\mathrm{x})=\mathrm{y}$ admet une unique solution $\mathrm{f}^{-1}(\mathrm{y})$. Or, quel que soit le couple $(\mathrm{a}, \mathrm{b})$ de ExE, $\mathrm{a} * \mathrm{~b}$ est élément de E. Donc, il existe une application $m$ de ExE dans $\mathrm{E}$ définie par $m(\mathrm{a}, \mathrm{b})=$ $\mathrm{f}^{-1}(\mathrm{a} * \mathrm{~b})$. De plus, comme a $\square \operatorname{Sup}(\mathrm{a}, \mathrm{b})$ et $\mathrm{b} \square \operatorname{Sup}(\mathrm{a}, \mathrm{b})$ avec $\operatorname{Sup}(\mathrm{a}, \mathrm{b}) \square \mathrm{E}$, on!a :

1. si l'ordre est compatible, alors $a \square b \square \operatorname{Sup}(a, b) \square \operatorname{Sup}(a, b)=f(\operatorname{Sup}(a, b))$ et comme $\mathrm{f}^{-1}$ est croissante, puisque f l'est, on!a : $\mathrm{f}^{\square 1}(\mathrm{a} \square \mathrm{b}) \square \mathrm{f}^{\square 1} \circ \mathrm{f}(\operatorname{Sup}(\mathrm{a}, \mathrm{b})=\operatorname{Sup}(\mathrm{a}, \mathrm{b})$, c'est-à-dire $m(\mathrm{a}, \mathrm{b}) \sqcap \operatorname{Sup}(\mathrm{a}, \mathrm{b})$;

2. si l'ordre est anticompatible, on trouve le même résultat puisque $\mathrm{f}^{\square 1}$ est alors décroissante. Même démonstration pour «Inf».

Reste à montrer que $m$ est croissante. Soit $\mathrm{a} \square \mathrm{b}$, par compatibilité on a pour tout $\mathrm{c}$ de $\mathrm{E} ! ! \mathrm{a} \square \mathrm{c} \square \mathrm{b} \square \mathrm{c}, \quad$ c'est-à-dire $\quad m(\mathrm{a}, \mathrm{c}) \square m(\mathrm{a}, \mathrm{c}) \square m(\mathrm{~b}, \mathrm{c}) \square m(\mathrm{~b}, \mathrm{c}) \quad$ ou encore $\mathrm{f}(m(\mathrm{a}, \mathrm{c})) \square \mathrm{f}(m(\mathrm{~b}, \mathrm{c}))$ et comme $\mathrm{f}^{-1}$ est croissante on en déduit que : $m(\mathrm{a}, \mathrm{c}) \square m(\mathrm{~b}, \mathrm{c})$.

COROLLAIRE 3.1 : Un magma réticulé $(\mathrm{E}, *, \leq)$ compatible (respectivement anticompatible) et fini est moyennable si, et seulement si, la loi * est idemfotente.

Preuve : D’après la proposition 5 ( $\$ 2.3$-b) et les théorèmes $1(\S 2.3-\mathrm{c})$ et 2 ( $\$ 2.3$-c).

COROLLAIRE 3.2 : Un magma réticulé $(\mathrm{E}, *, \leq)$ compatible (respectivement anticompatible) avec $\leq$ un ordre total, est moyennable si, et seulement si, la loi $*$ est idemfotente.

Preuve : D'après la proposition $4(\S 2.3-b)$ et les théorèmes $1(\S 2.3-c)$ et $2(\S 2.3-c)$.

COROLLAIRE 3.3 : Un magma réticulé $(\mathrm{E}, *, \leq)$ compatible (respectivement anticompatible) est moyennable selon la loi $*$ si, et seulement si, la loi $*$ est idempotente.

Preuve : Dans ce cas $\mathrm{f}=i d_{\mathrm{E}}$. Si la loi est compatible, c'est évident. Et si la loi est à la fois idempotente et anticompatible on a alors $\operatorname{Sup}(a, b) !=! \operatorname{Inf}(a, b)$, car : $\operatorname{Sup}(a, b) ! \square ! \operatorname{Inf}(a, b) * \operatorname{Sup}(a, b)$ et $\operatorname{Inf}(a, b) * \operatorname{Sup}(a, b) \square \operatorname{Inf}(a, b)$, et la seule relation d'ordre possible est l'égalité.

Naturellement, on peut trouver des structures non compatibles (ni anti-compatibles) mais moyennables. Examinons l'exemple suivant :

Considérons l'ensemble $\mathbb{N}$ des entiers naturels (zéro compris). Pour tout entier a, on montre qu'il existe un unique couple d'entiers $\left(a_{1}, a_{2}\right)$ tel que $a=2^{a_{1}}\left(2 a_{2}+1\right)$. Munissons $\mathbb{N}$ de l'ordre «ঝ» total défini par :

$\mathrm{a} \prec b \square\left\{\mathrm{a}_{1}<\mathrm{b}_{1}\right.$ ou $\left(\mathrm{a}_{1}=\mathrm{b}_{1}\right.$ et $\left.\left.\mathrm{a}_{2} \square \mathrm{b}_{2}\right)\right\}$ et de la loi idempotente définie par : $\mathrm{a} \square \mathrm{b}=2^{\max \left(\mathrm{a}_{1}, \mathrm{~b}_{1}\right)}\left(2 \min \left(\mathrm{a}_{2}, \mathrm{~b}_{2}\right)+1\right)$, où max et min sont définis selon l'ordre usuel des entiers. 
Cette loi n'est pas compatible avec l'ordre, car par exemple on a bien $44 \prec 40$ (puisque $44=2^{2}(2 \square 5+1)$ et $40=2^{3}(2 \square 2+1)$ ) mais $80=40 \square 112 \prec 44 \square 112=112$ car, $80=2^{4}(2 \square 2+1)$ et $112=2^{4}(2 \square 3+1)$. Elle n'est pas non plus anticompatible, car $3 \prec 56 \square 2=3 \square 2 \prec 56 \square 2=8$. Pourtant, «*» est bien moyenne selon elle-même, car on vérifie aisément que si $\mathrm{a} \prec \mathrm{b}$, on a toujours $\mathrm{a} \prec \mathrm{a} \square \mathrm{b} \prec \mathrm{b}$.

\section{d) Cas des structures finies}

En sciences humaines, on est souvent amené à étudier des structures finies, qui par ailleurs, opèrent fréquemment en dehors du champ numérique pour ne conserver que le caractère ordinal, voire opératoire des éléments mis en jeu. Aussi, examinons plus particulièrement le cas des structures moyennables finies, susceptibles de s'affranchir de l'emprise numérique.

Malheureusement, certaines exigences et non des moindres, que réclame l'usage des moyennes, s'accommodent assez mal des constructions algébriques et ordinales dans le domaine discret et fini. En particulier, celles-ci ne respectent généralement pas la monotonie de la moyenne, ni même ne permettent une généralisation à plusieurs éléments. Une des raisons de cet échec provient de l'absence de densité que présentent, naturellement, les ensembles discrets et finis. Ainsi, par exemple, pour parvenir à construire des structures finies totalement ordonnées qui respectent la règle de la valeur intermédiaire, il faut utiliser des lois de composition qui par nature ne sont généralement ni associatives, ni compatibles (respectivement anticompatible) avec la relation d'ordre total. En fait, comme nous allons le montrer dans le cadre des ordres totaux, les seules lois de composition qui donnent pleinement satisfaction sont des lois idempotentes, autrement dit des moyennes selon elles-mêmes.

Commençons par examiner une des structures les plus fondamentales en algèbre, celle de groupe, qui dans le cas fini conduit toujours à un échec.

PROPOSITION 3.6 : Hormis le groupe d'ordre un, il n'existe aucun groupe réticulé fini moyennable.

Preuve : Soit un groupe fini réticulé $(\mathrm{G}, *, \leq)$, avec $|\mathrm{G}|>1$. Montrons par l'absurde que $(\mathrm{G}, *, \leq)$ n'est pas moyennable.

Si la loi $*$ n'est pas idemfotente, le groupe n'est pas moyennable ; donc nous supposerons qu'elle l'est. Par ailleurs, on peut supposer $|\mathrm{G}|>2$, car il n'existe qu'un seul groupe d'ordre deux, et sa la loi n'est pas idemfotente (en revanche celui d'ordre 3 en possède une $)^{4}$. Considérons, alors, la loi $\oplus$ définie sur $\mathrm{G}$ par : $\mathrm{a} \oplus \mathrm{b}=\mathrm{f}^{\square 1}(\mathrm{a} \square \mathrm{b})$ où $\mathrm{f}^{\square 1}$ est la bijection définie sur $\mathrm{G}$ par : $\mathrm{f}(\mathrm{x})=\mathrm{x} \square \mathrm{x}$. La loi $\oplus$ est naturellement idempotente et la structure $(\mathrm{G}, \oplus)$ est un quasi-groupe (i.e. une structure qui n'est pas un groupe mais dont tous les éléments sont réguliers). En effet, tous les éléments sont réguliers pour $\oplus$ (carré latin) en vertu de ce qu'ils le sont pour la loi $*$ du groupe $(\mathrm{G}, *)$ d'après la proposition $5(\S 2.2-\mathrm{c})$; et $(\mathrm{G}, \oplus)$ ne peut pas avoir une structure de groupe, car nécessairement $\oplus$ n'est pas associative d'après la proposition $6(\S 2.2-\mathrm{c})$.

\footnotetext{
${ }^{4}$ Pour les groupes finis abéliens, on possède un critère. En effet, tout groupe abélien fini G est isomorphe à!: $\frac{\mathbb{Z}}{\mathrm{n}_{1}} \square \frac{\mathbb{Z}}{\mathrm{n}_{2}} \ldots \square \frac{\mathbb{Z}}{\mathrm{n}_{\mathrm{k}} \mathbb{Z}}$ avec $n_{i}=p_{i}^{k_{i}}$ pour $p_{i}$ premiers non nécessairement distincts. Alors, la loi du groupe est idemfotente si et seulement si $: p_{i} \neq 2$ pour tout $i$. (preuve par lemme des restes chinois).
} 
Maintenant, supposons $(\mathrm{G}, *, \leq)$ moyennable ; d'après le théorème $1(\S 2.3-\mathrm{c})$, sa moyenne est $\oplus$. Posons $x_{0}=\operatorname{Inf}(G)$, comme $(G, \oplus)$ est un quasi-groupe, on a nécessairement pour tout $\mathrm{x} \square \mathrm{G} \square \mathrm{x}_{0} \quad: \mathrm{f}^{\square 1}\left(\mathrm{x}_{0} \square \mathrm{x}\right) \neq \mathrm{x}_{0}$. Soit $\mathrm{x}_{1} \square \mathrm{G} \square \mathrm{x}_{0}$, il existe car $|\mathrm{G}|>2$, et par hypothèse : $\operatorname{Inf}\left(\mathrm{x}_{0}, \mathrm{x}_{1}\right) \square \mathrm{f}^{\square 1}\left(\mathrm{x}_{0} \square \mathrm{x}_{1}\right) \square \operatorname{Sup}\left(\mathrm{x}_{0}, \mathrm{x}_{1}\right)$, c'est-à-dire : $\mathrm{x}_{0}<\mathrm{f}^{\square 1}\left(\mathrm{x}_{0} \square \mathrm{x}_{1}\right) \square \mathrm{x}_{1}$. Mais, $\mathrm{f}^{\square 1}\left(\mathrm{x}_{0} \square \mathrm{x}_{1}\right) \neq \mathrm{x}_{1}$ car sinon on aurait : $\mathrm{f}^{\square 1}\left(\mathrm{x}_{0} \square \mathrm{x}_{1}\right)=\mathrm{x}_{1}=\mathrm{f}^{\square 1}\left(\mathrm{x}_{1} \square \mathrm{x}_{1}\right)$, c'est-à-dire : $\mathrm{x}_{0} \square \mathrm{x}_{1}=\mathrm{x}_{1} \square \mathrm{x}_{1}$ et par régularité $\mathrm{x}_{0}=\mathrm{x}_{1}$, ce qui est contraire à l'hypothèse. Ainsi, en posant : $x_{k}=f^{\square 1}\left(x_{0} \square x_{k-1}\right)$, on peut exhiber la suite d'inégalités strictes suivante : $\mathrm{x}_{0}<\mathrm{f}^{\square 1}\left(\mathrm{x}_{0} \square \mathrm{x}_{\mathrm{k}}\right)<\cdots<\mathrm{f}^{\square 1}\left(\mathrm{x}_{0} \square \mathrm{x}_{1}\right)<\mathrm{x}_{1}$. Or $\mathrm{G}$ est fini, donc il existe un entier $\mathrm{k}$ tel que $\mathrm{x}_{\mathrm{k}}=\mathrm{x}_{1}$, ce qui contredit la relation précédente.

Illustrons ce résultat par un exemple :

Soit $\mathrm{G}=\{\mathrm{a}, \mathrm{b}, \mathrm{c}, \mathrm{d}, \mathrm{e}\}$ ordonné totalement selon l'ordre lexicographique. Le seul groupe d'ordre 5, dont la loi $*$ est idemfotente, est le groupe dont la table de composition est donnée par : (à droite on a représenté celle de la loi $\oplus$ associée).

\begin{tabular}{cccccc}
\hline $\mathrm{C}$ & $\mathrm{a}$ & $\mathrm{b}$ & $\mathrm{c}$ & $\mathrm{d}$ & $\mathrm{e}$ \\
\hline $\mathrm{a}$ & $\mathrm{a}$ & $\mathrm{b}$ & $\mathrm{c}$ & $\mathrm{d}$ & $\mathrm{e}$ \\
$\mathrm{b}$ & $\mathrm{b}$ & $\mathrm{c}$ & $\mathrm{d}$ & $\mathrm{e}$ & $\mathrm{a}$ \\
$\mathrm{c}$ & $\mathrm{c}$ & $\mathrm{d}$ & $\mathrm{e}$ & $\mathrm{a}$ & $\mathrm{b}$ \\
$\mathrm{d}$ & $\mathrm{d}$ & $\mathrm{e}$ & $\mathrm{a}$ & $\mathrm{b}$ & $\mathrm{c}$ \\
$\mathrm{e}$ & $\mathrm{e}$ & $\mathrm{a}$ & $\mathrm{b}$ & $\mathrm{c}$ & $\mathrm{d}$ \\
\hline
\end{tabular}

\begin{tabular}{cccccc}
\hline$\oplus$ & $\mathrm{a}$ & $\mathrm{b}$ & $\mathrm{c}$ & $\mathrm{d}$ & $\mathrm{e}$ \\
\hline $\mathrm{a}$ & $\mathrm{a}$ & $\mathrm{d}$ & $\mathrm{b}$ & $\mathrm{e}$ & $\mathrm{c}$ \\
$\mathrm{b}$ & $\mathrm{d}$ & $\mathrm{b}$ & $\mathrm{e}$ & $\mathrm{c}$ & $\mathrm{a}$ \\
$\mathrm{c}$ & $\mathrm{b}$ & $\mathrm{e}$ & $\mathrm{c}$ & $\mathrm{a}$ & $\mathrm{d}$ \\
$\mathrm{d}$ & $\mathrm{e}$ & $\mathrm{c}$ & $\mathrm{a}$ & $\mathrm{d}$ & $\mathrm{b}$ \\
$\mathrm{e}$ & $\mathrm{c}$ & $\mathrm{a}$ & $\mathrm{d}$ & $\mathrm{b}$ & $\mathrm{e}$ \\
\hline
\end{tabular}

On a bien pour tout $\mathrm{x}$ et $\mathrm{y}$ de $\mathrm{G}, \mathrm{x} \oplus \mathrm{y}=\mathrm{f}^{\square 1}(\mathrm{x} \square \mathrm{y})$ avec $\mathrm{f}$ bijective : $\mathrm{f}(\mathrm{x})=\mathrm{x} \square \mathrm{x}$; mais par exemple : $\mathrm{a} \oplus \mathrm{b}=\mathrm{d}$ avec $\mathrm{d}>\operatorname{Sup}(\mathrm{a}, \mathrm{b}) !=! \mathrm{b}$.

Exploitons le théorème de caractérisation qui, en réalité, n'est rien d'autre que la contraction des deux axiomes de la définition de la moyenne, pour étudier les structures finies. Pour qu'une structure $(\mathrm{E}, *, \leq)$ soit moyennable, la table de composition de la loi!* doit nécessairement satisfaire la contrainte suivante : pour tout a et $\mathrm{b}$ éléments de! $\mathrm{E}$, $\square^{\square 1}(\mathrm{a} \square \mathrm{b}) \square\{\mathrm{x} \square \mathrm{E}, \operatorname{Inf}(\mathrm{a}, \mathrm{b}) \square \mathrm{x} \square \operatorname{Sup}(\mathrm{a}, \mathrm{b})\}$ où $\square$ est une bijection de E. Examinons, dans certains cas, les particularités que présentent ces tables de composition.

Étudions le cas particulier important où $(\mathrm{E}, *, \leq)$ est une structure finie totalement ordonnée et posons : $|\mathrm{E}|=\mathrm{n}$.

Notons $\left(\square_{i, j}\right)$ la matrice carrée associée à la table de composition, dont les éléments $x_{i}$ de $\mathrm{E}$ sont rangés selon l'ordre de la chaîne couvrante maximale : $x_{1} \square x_{2} \square \cdots \square x_{n}$. On pose donc $\square_{i, j}=x_{i} \square x_{j}$.

Notons $[i]$ la matrice extraite de la matrice $\left(\square_{i, j}\right)$ ayant pour première colonne, la colonne $i$, et pour dernière ligne, la ligne $i$; et désignons par $\mathrm{E}_{i}$ l'ensemble des éléments de $\mathrm{E}$ figurant dans l'une, au moins, des deux matrices $[i]$ et sa transposée ${ }^{t}[i]$, c'est-à-dire l'ensemble : $\mathrm{E}_{i}=\left\{x \square \mathrm{E}, x \square[i] \square{ }^{t}[i]\right\}$. 
Enfin, notons $\left[\overline{i]}\right.$ et ${ }^{c} \overline{[i]}$ les deux matrices carrées extraites de $\left(\square_{i, j}\right)$, respectivement définies par: $\square_{p, q} \square\left[\overline{i]}\right.$ pour tout $p<i$ et $q<i$, et $\square_{p, q} \square^{c} \overline{[i]}$ pour tout $p>i$ et $q$ $>i$ !; et désignons par $\overline{\mathrm{E}}_{i}$ l'ensemble des éléments de $\mathrm{E}$ figurant dans l'une, au moins, des deux matrices $\overline{[i]}$ et ${ }^{c} \overline{[i]}$ c'est-à-dire l'ensemble : $\overline{\mathrm{E}}_{i}=\left\{x \square \mathrm{E}, x \square \overline{[i]} \square^{c} \overline{[i]}\right\}$.

Soit $\square$ une permutation des indices : $\square \square \square_{n}$, (donc associée à une bijection de $\left.\mathrm{E}\right)$. On définit la loi de composition «*» dans $\mathrm{E}$ en construisant sa table de la façon suivante!: pour tout $i \square\{1, \cdots, n\}, x_{i} \square x_{i}=x_{\square(i)}$ et pour tous les éléments non diagonaux on complète la table par des éléments de $\mathrm{E}$, avec pour seule contrainte : pour tout $i \square\{1, \cdots, n\}, \quad x_{\square(i)} \square \overline{\mathrm{E}}_{i}$.

Toute table de composition construite de cette manière est dite : carré moyennable, dans la mesure où justement $(\mathrm{E}, *, \leq)$ est moyennable, comme le précise le théorème suivant :

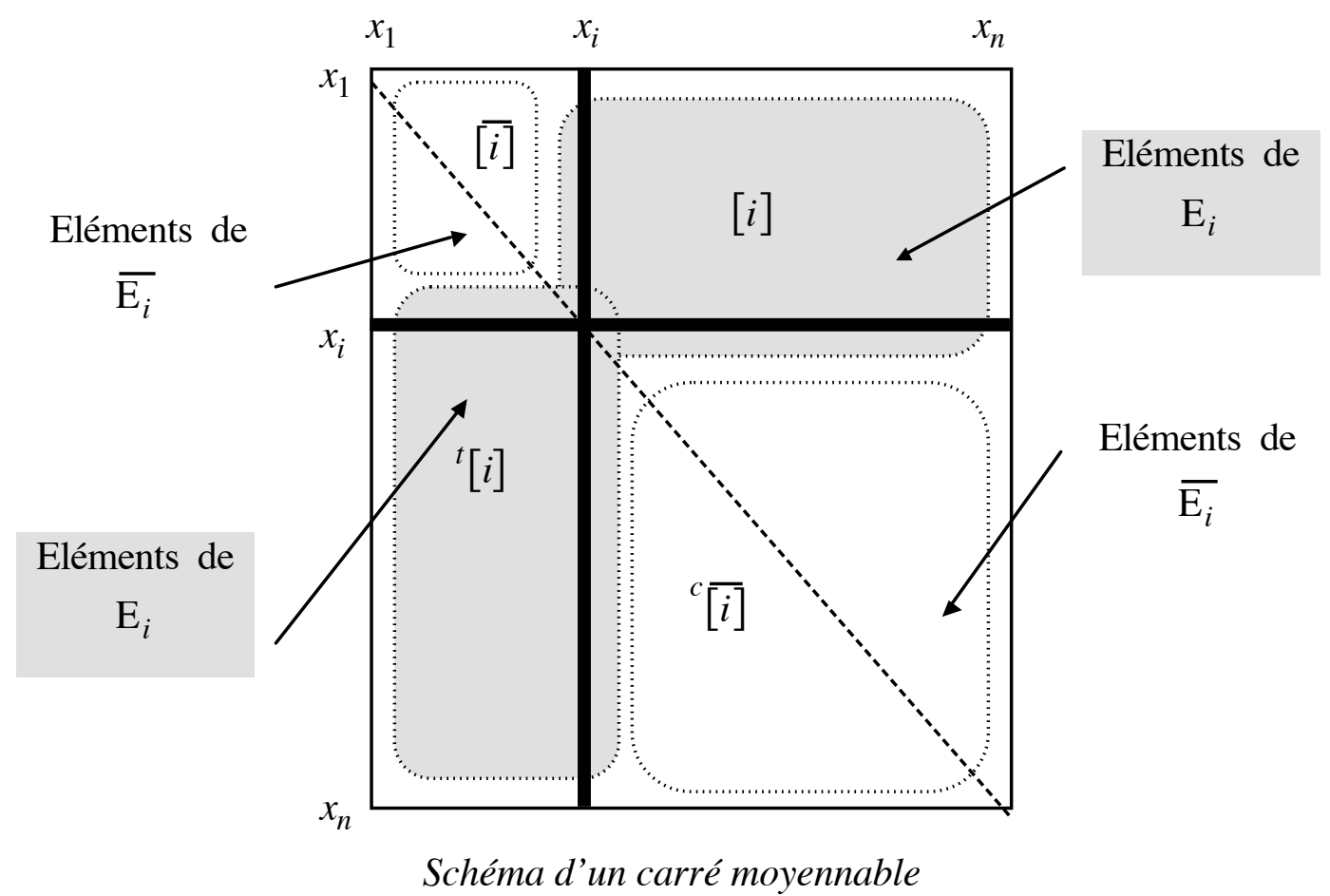

THÉORÈME 3.3 : Soit $(\mathrm{E}, *, \leq)$ un magma fini totalement ordonné. $(\mathrm{E}, *, \leq)$ est moyennable si, et seulement si, la table de composition de $*$ est un carré moyennable.

Preuves : ( $\square$ ) Supposons $(\mathrm{E}, *, \leq)$ moyennable. En vertu du théorème 1 ( $\$ 2.3-\mathrm{c})$, la loi!* est idemfotente. Par conséquent, il existe une permutation $\square$ des indices telle que pour tout $i \square\{1, \cdots, n\}, \quad x_{i} \square x_{i}=x_{\square(i)}$. Soit maintenant : $i ! \neq ! j$, il existe un entier $k$ tel que $x_{i} \square x_{j}=x_{k}$, or puisque $(\mathrm{E}, *, \leq)$ est moyennable on a : $m\left(x_{i}, x_{j}\right)=x_{\square^{\square 1}(k)}$ avec $\operatorname{Inf}\left(x_{i}, x_{j}\right) \square x_{\square^{\square 1}(k)} \square \operatorname{Sup}\left(x_{i}, x_{j}\right)$. Or, pour tout entier $l$ tel que $\operatorname{Inf}(i, j) \square l \square \operatorname{Sup}(i, j)$, on a par définition des matrices $[l]: x_{i} \square x_{j}=x_{k} \square[l] \square{ }^{t}[l]$ donc en particulier pour 
$l=\square^{\square 1}(k)$ et par suite $x_{k} \square \mathrm{E}_{\square^{\square 1}(k)}$. Montrons, maintenant que $x_{k} \square \overline{\mathrm{E}_{\square^{\square 1}(k)}}$. Supposons le contraire, on aurait ou bien $x_{k} \square\left[\square^{\square 1}(k)\right]$ ou bien $x_{k} \square\left[\square^{\square 1}(k)\right]$. Si $x_{k} \square\left[\square^{\square 1}(k)\right]$, alors il existerait deux entiers $p$ et $q$ strictement inférieurs à $\square^{\square 1}(k)$ tel que $x_{p} \square x_{q}=x_{k}$, et par suite $\operatorname{Sup}\left(x_{p}, x_{q}\right)<x_{\square^{\square !}(k)}$; or c'est absurde car $x_{\square^{\square !}(k)}=m\left(x_{p}, x_{q}\right) \square \operatorname{Sup}\left(x_{p}, x_{q}\right)$.

De même, si $x_{k} \square^{c}\left[\square^{\square 1}(k)\right]$, il existerait deux entiers $p$ et $q$ strictement supérieurs à $\square^{\square 1}(k)$ tel que $x_{p} \square x_{q}=x_{k}$, et par suite $x_{\square^{\square}(k)}<\operatorname{Inf}\left(x_{p}, x_{q}\right)$; ce qui est absurde puisque $\operatorname{Inf}\left(x_{p}, x_{q}\right) \square m\left(x_{p}, x_{q}\right)=x_{\square^{\square}(k)}$. La table de la loi $*$ est donc bien nécessairement un carré moyennable dès lors que $(\mathrm{E}, *, \leq)$ est moyennable.

( $\square$ ) Réciproquement, soit une loi $*$ définie à partir d'un carré moyennable, montrons que $(\mathrm{E}, *, \leq)$ est moyennable. Si $i=j$, c'est clair. Soit $i \neq j$, on peut supposer $i<j$, car même si les matrices $[i]$ et ${ }^{t}[i]$ ne sont pas symétriques (la loi $*$ n'est pas nécessairement commutative) elles jouent un rôle symétrique dans le raisonnement. Ainsi, il $k$ tel que : $x_{i} \square x_{j}=x_{k}$ et puisque $\square$ est bijective, il existe aussi un unique entier $\square^{\square 1}(k)$ tel que $x_{\square^{\square}(k)} \square x_{\square^{\square 1}(k)}=x_{k}$. Montrons que nécessairement $i \square \square^{\square 1}(k) \square j$. En effet, si $\square^{\square 1}(k)<i$, on aurait, puisque $x_{k}=\square_{i, j}, x_{k} \square \overline{\mathrm{E}_{\square^{\square 1}(k)}}$ ce qui est contraire à l'hypothèse. De même, si $j<\square^{\square 1}(k)$, on aurait, de nouveau : $x_{k} \square \overline{\mathrm{E}_{\square^{\square 1}(k)}}$. Par conséquent!: $i \square \square^{\square 1}(k) \square j$ et par suite $x_{i} \square x_{\square^{\square 1}(k)} \square x_{j}$, donc, $m\left(x_{i}, x_{j}\right)=x_{\square^{\square 1}(k)}$.

\section{Illustration}

Soit $\mathrm{E}=\{\mathrm{a}, \mathrm{b}, \mathrm{c}, \mathrm{d}, \mathrm{e}\}$ ordonné totalement selon l'ordre lexicographique et dont la table de composition de $*$ est donnée par (à droite on a représenté celle de la moyenne) :

\begin{tabular}{cccccc}
\hline $\mathrm{d}$ & $\mathrm{a}$ & $\mathrm{b}$ & $\mathrm{c}$ & $\mathrm{d}$ & $\mathrm{e}$ \\
\hline $\mathrm{a}$ & $\mathrm{d}$ & $\mathrm{a}$ & $\mathrm{a}$ & $\mathrm{d}$ & $\mathrm{e}$ \\
$\mathrm{b}$ & $\mathrm{d}$ & $\mathrm{a}$ & $\mathrm{a}$ & $\mathrm{a}$ & $\mathrm{b}$ \\
$\mathrm{c}$ & $\mathrm{e}$ & $\mathrm{a}$ & $\mathrm{e}$ & $\mathrm{e}$ & $\mathrm{c}$ \\
$\mathrm{d}$ & $\mathrm{d}$ & $\mathrm{e}$ & $\mathrm{c}$ & $\mathrm{c}$ & $\mathrm{c}$ \\
$\mathrm{e}$ & $\mathrm{c}$ & $\mathrm{c}$ & $\mathrm{e}$ & $\mathrm{b}$ & $\mathrm{b}$ \\
\hline
\end{tabular}

\begin{tabular}{cccccc}
\hline$m$ & $\mathrm{a}$ & $\mathrm{b}$ & $\mathrm{c}$ & $\mathrm{d}$ & $\mathrm{e}$ \\
\hline $\mathrm{a}$ & $\mathrm{a}$ & $\mathrm{b}$ & $\mathrm{b}$ & $\mathrm{a}$ & $\mathrm{c}$ \\
$\mathrm{b}$ & $\mathrm{a}$ & $\mathrm{b}$ & $\mathrm{b}$ & $\mathrm{b}$ & $\mathrm{e}$ \\
$\mathrm{c}$ & $\mathrm{c}$ & $\mathrm{b}$ & $\mathrm{c}$ & $\mathrm{c}$ & $\mathrm{d}$ \\
$\mathrm{d}$ & $\mathrm{a}$ & $\mathrm{c}$ & $\mathrm{d}$ & $\mathrm{d}$ & $\mathrm{d}$ \\
$\mathrm{e}$ & $\mathrm{d}$ & $\mathrm{d}$ & $\mathrm{c}$ & $\mathrm{e}$ & $\mathrm{e}$ \\
\hline
\end{tabular}

Il est clair que toute autre loi, obtenue par une quelconque permutation des composés de la loi $*$, admet la même moyenne $m$. (Voir supra proposition 1 ( $§ 2.4$ ). En particulier, il existe une unique permutation $\square$ de $E$ telle que pour tout a et $b$ de!!E, on $\mathrm{a}: \square(\mathrm{a} \square \mathrm{b})=m(\mathrm{a}, \mathrm{b})$.

Dans le cas d'un ensemble fini totalement ordonné, il est facile de caractériser toutes les structures moyennables et même de les dénombrer. En effet, pour construire un carré moyennable (et donc une structure finie totalement ordonnée moyennable) il faut commencer par se donner une bijection de $\mathrm{E}$; si $|\mathrm{E}|=\mathrm{n}$, il y a donc (n!) choix possibles. Puis, pour chaque bijection $\square$ choisie, il faut affecter aux $\mathrm{n}(\mathrm{n}-1)$ couples $(i, j)$ restants $(i \neq j)$ de la table de composition, un unique élément de $\mathrm{E}$, choisi de sorte qu'il ne déroge 
pas à la règle de construction des carrés moyennables. Pour chaque couple, plusieurs solutions sont possibles ; précisons cela en notant $E_{i, j}$ l'ensemble de tous les éléments de E susceptibles de valoir $x_{i} \square x_{j}$ dans une table de carré moyennable relative à la bijection $\square$. Nous dirons qu'un éléments de $E_{i, j}$ est un candidat admissible pour le couple $(i, j)$.

THÉORÈME 3.4 : Soit $(\mathrm{E}, \leq)$ une structure finie totalement ordonnée de cardinal $\mathrm{n}$.

Désignons par : $x_{1} \square x_{2} \square \cdots \square x_{n}$ sa chaîne couvrante maximale. Tout magma (E,*, $\left.\leq\right)$ totalement ordonné et fini est moyennable si, et seulement si, sa loi de composition * vérifie la relation!:

pour tout $i \square\{1, \cdots, n\}$ et pour tout $j \square\{1, \cdots, n\}$, on a!:

$x_{i} \square x_{j} \square E_{i, j}$ avec $E_{i, j}=\bigcup_{k=i}^{k=j}\left\{\square\left(x_{k}\right)\right\}$ où $\square$ est une bijection de E.

Preuves: ( $\bigsqcup$ ) Supposons $(\mathrm{E}, *, \leq)$ moyennable. En vertu du théorème $1(\S 2.3-\mathrm{c})$, la loi!* est idemfotente. Donc, il existe une bijection $\square$ de $\mathrm{E}$ telle que pour tout $i \square\{1, \cdots, n\}$ on $\mathrm{a}: x_{i} \square x_{i}=\square\left(x_{i}\right)$. D'autre part, d'après le théorème $3(\S 2.3-\mathrm{d})$, la table de composition de * est nécessairement un carré moyennable. Par conséquent, tout élément $x$ de $\mathrm{E}$ est un candidat admissible pour le couple $(1, \mathrm{n})$; en effet, $x_{1} \square x_{n} \square \bigcap_{k=1}^{k=n} \mathrm{E}_{k}$ donc si $x=x_{1} \square x_{n}$, il existe $\square(x)$ tel que $x \square \overline{\mathrm{E}_{\square(x)}}$. Finalement, $E_{1, n}=\bigcup_{k=1}^{\substack{k=n \\ k=1}}\left\{\square\left(x_{k}\right)\right\}=\mathrm{E}$. Raisonnons, maintenant, par récurrence!; pour tout entier $p$ tel que $1 \square p \square n$, supposons que $\quad E_{1, p}=\bigcup_{k=1}^{k=p}\left\{\square\left(x_{k}\right)\right\}$ et montrons que $E_{1, p \square 1}=\bigcup_{k=1}^{k=p \square 1}\left\{\square\left(x_{k}\right)\right\}, \quad$ c'est-à-dire $\square\left(x_{p}\right) \square E_{1, p \square 1}$. Or, $\square\left(x_{p}\right) \square \overline{\mathrm{E}_{p}}$, il n'est donc pas un élément de la matrice carrée $\square\left(x_{p}\right)$. Mais comme $x_{p \square 1}<x_{p}$, tout élément de $E_{1, p \square 1}$ est un élément de la matrice $\left.\square\left(x_{p}\right)\right]$, d'où nécessairement $\square\left(x_{p}\right) \square E_{1, p \square 1}$. Le même raisonnement nous conduit à $E_{2, p}=\bigcup_{k=2}^{k=p}\left\{\square\left(x_{k}\right)\right\}$ et plus généralement, pour tout $i$ et $j$ inférieurs à $\mathrm{n}$, on $\mathrm{a}: E_{i, j}=\bigcup_{k=i}^{k=j}\left\{\square\left(x_{k}\right)\right\}$.

( $\square$ ) La réciproque est immédiate ; puisque, si pour tout $i \square\{1, \cdots, n\}$ et pour tout $j \square\{1, \cdots, n\}$, on a : $x_{i} \square x_{j} \square E_{i, j}$ avec $E_{i, j}=\bigcup_{k=i}^{k=j}\left\{\square\left(x_{k}\right)\right\}$ où $\square$ est une bijection de $\mathrm{E}$, alors la table de $*$ est un carré moyennable.

COROLLAIRE 3.4 : Soit $(\mathrm{E}, \leq)$ une structure finie totalement ordonnée. Posons $|\mathrm{E}|=$ n. Le nombre de magma totalement ordonné, fini et moyennable est : $(n !) \square_{k=2}^{k=n} k^{2(n \square k+1)}$. 
Preuve : Pour toute bijection $\square$ fixée de E, on a $E_{i, j}=\bigcup_{k=i}^{k=j}\left\{\square\left(x_{k}\right)\right\}$ et donc $\left|E_{i, j}\right|=|i \square j+1|$. Or, pour tout entier $k \square n$, on a $\left|E_{k, n}\right|=\left|E_{p, n \square k+p}\right|$ pour tout $1 \square p \square k$. Il y a donc $k$ ensembles d'éléments admissibles, tous de même cardinal égal à $\left|E_{k, n}\right|=n \square k+1$. Mais comme pour tout $i \square\{1, \cdots, n\}$ et pour tout $j \square\{1, \cdots, n\}$, on a $E_{i, j}=E_{j, i}$, on dénombre pour chaque $k$ variant de 1 à $\mathrm{n}, 2 k$ ensembles d'éléments admissibles, tous de cardinal égal à $n \square k+1$. Comme il y a $\left|E_{i, j}\right|$ façons de choisir un élément $x_{i} \square x_{j}$, il y a donc $\left[\left|E_{1, n}\right| \square\left|E_{2, n}\right|^{2} \square\left|E_{3, n}\right|^{\beta} \square \cdots \square\left|E_{n, n}\right|^{n}\right]^{2}$ façons de remplir une table de composition de carré moyennable pour une bijection $\square$ donnée ; c'est-à-dire $\left[n \square(n \square 1)^{2} \square(n \square 3)^{3} \square \cdots \square 1^{n}\right]^{2}$ ce qui peut encore s'écrire $\square_{k=2}^{k=n} k^{2(n \square k+1)}$. Et comme il existe (n!) bijections possibles, on a le résultat.

Dans le dernier exemple cité ci-dessus, on a pu remarquer que la loi $*$ n'était ni associative, ni compatible (respectivement anti-compatible) avec la relation d'ordre total. Nous allons préciser ce fait.

THÉORÈME 3.5 : Soit $(\mathrm{E}, *, \leq)$ un magma fini, totalement ordonné et moyennable. Si la loi $*$ n'est pas idempotente, alors elle n'est pas associative.

Preuve : Par l'absurde, supposons la loi $*$ associative. Désignons par!: $x_{1} \square x_{2} \square \cdots \square x_{n}$ la chaîne couvrante maximale de E. Puisque la loi $*$ n'est pas idempotente, il existe une permutation $\square$ de $\mathrm{E}$ telle que $\square \neq i d_{\mathrm{E}}$ et pour tout entier $k \square n, \square\left(x_{k}\right)=x_{k} \square x_{k}$. Il existe donc un entier $i$ tel que $\square\left(x_{i}\right) \neq x_{i}$ et tel que pour tout entier $k>i$, on a $\square\left(x_{k}\right)=x_{k}$.

Posons alors, pour cet entier $i$ !: $y=\square\left(x_{i}\right)$ et $z=\square^{\square 1}\left(x_{i}\right)$.

Montrons d'abord que $z<x_{i}$. Si cela n'était pas le cas, on aurait: ou bien $z=x_{i}$ et dans ce cas $\square(z)=\square\left(x_{i}\right)$, c'est-à-dire $y=x_{i}$ ce qui est contraire à l'hypothèse ; ou bien $z>x_{i}$ et dans ce cas, par hypothèse, on a $\square(z)=z$ ce qui conduit à l'absurdité $z=x_{i}$.

Maintenant, utilisons la propriété d'associativité, nous avons : $(z \square z) \square x_{i}=z \square\left(z \square x_{i}\right)$, c'est-à-dire $x_{i} \square x_{i}=z \square\left(z \square x_{i}\right)$ ou encore $y=z \square\left(z \square x_{i}\right)$.

Comme l'ordre est total, de deux choses l'une!: ou bien $z \square x_{i}<x_{i}$, ou bien $z \square x_{i} \geq x_{i}$.

Supposons $z \square x_{i}<x_{i}$. Comme $(\mathrm{E}, *, \leq)$ est un magma fini, totalement ordonné et moyennable, la table de $*$ est un carré moyennable d'après le théorème 3 ( $\$ 2.3-\mathrm{d})$; par conséquent puisque $z<x_{i}$, pour tout $x$ de $\mathrm{E}$ tel que $x<x_{i}$ on a nécessairement $z \square x \neq \square\left(x_{i}\right)$, donc $z \square x \neq y$ et en particulier pour $x=z \square x_{i}$, on a $z \square\left(z \square x_{i}\right) \neq y$ ce qui contredit la propriété d'associativité ci-dessus.

Supposons $z \square x_{i} \geq x_{i}$. E étant fini, il existe un entier tel que $z \square x_{i}=x_{k}$. Deux cas se présentent $k=i$ ou $k>i$. Si $k=i$, alors $z \square x_{i}=x_{i}$ et par suite $z \square\left(z \square x_{i}\right)=x_{i}$ et d'où par associativité $y=x_{i}$ ce qui est contraire à l'hypothèse. Si 
$k>i$, comme $z<x_{i}$ et $x_{i}<x_{k}$ on a nécessairement $z \square x_{i} \square \overline{\mathrm{E}_{k}}$. Cependant, $z \square x_{i}=x_{k}=\square\left(x_{k}\right)$ et donc $\square\left(x_{k}\right) \square \overline{\mathrm{E}_{k}}$ ce qui est impossible dans un carré moyennable.

Dans tous les cas, nous aboutissons à une contradiction, la loi $*$ ne peut-être associative.

THÉORÈME 3.6 : Soit $(\mathrm{E}, *, \leq)$ un magma fini, totalement ordonné et moyennable. Si la loil* n'est pas idempotente, alors elle n'est pas compatible avec la relation d'ordre total.

Preuve : Par l'absurde, supposons la loi * compatible avec la relation d'ordre total. Désignons par!: $x_{1} \square x_{2} \square \cdots \square x_{n}$ la chaîne couvrante maximale de E. Puisque la loi $*$ n'est pas idempotente, il existe une permutation $\square$ de $\mathrm{E}$ telle que $\square \neq i d_{\mathrm{E}}$ et pour tout entier $k \square n, \square\left(x_{k}\right)=x_{k} \square x_{k}$. Il existe donc un entier $i$ tel que $\square\left(x_{i}\right) \neq x_{i}$ et tel que pour tout entier $k>i$, on a $\square\left(x_{k}\right)=x_{k}$.

Posons alors, pour cet entier $i$ !: $y=\square\left(x_{i}\right)$ et $z=\square^{\square 1}\left(x_{i}\right)$.

Montrons d'abord que $z<x_{i}$. Si cela n'était pas le cas, on aurait!! !ou bien $z=x_{i}$ et dans ce cas $\square(z)=\square\left(x_{i}\right)$, c'est-à-dire $y=x_{i}$ ce qui est contraire à l'hypothèse ; ou bien $z>x_{i}$ et dans ce cas, par hypothèse, on a $\square(z)=z$ ce qui conduit à l'absurdité $z=x_{i}$.

Montrons également que $y<x_{i}$. Par définition on a!: $y \neq x_{i}$; supposons que $x_{i}<y$, on aurait $\square(y)=y$ par hypothèse, et $\square\left(x_{i}\right)=y$ par définition. Comme $\sqcup$ est injective, on en déduirait que $y=x_{i}$, ce qui est absurde.

Puisque la loi $*$ est supposée compatible avec la relation d'ordre total, la bijection $\sqcup$ est croissante d'après la proposition $2(\S 2.3-\mathrm{a})$ et même strictement croissante puisque l'ordre est total et que $\sqcup$ est injective. Ainsi, de $z<x_{i}$ on tire $\square(z)<\square\left(x_{i}\right)$ c'est-à-dire $x_{i}<y$, ce qui, comme nous venons de le voir, est impossible.

e) Exemple d'une structure finie partiellement ordonnée et moyennable

\section{DÉFINITION : Fuseau}

On appelle Fuseau, un treillis (F, $\leq$ ) fini pour lequel l'infimum et le supremum de toute paire d'éléments incomparables sont respectivement égaux à l'infimum et au supremum de F. C'est-à-dire, pour tout $x$ et y de F tel que $x ! \square y$, on a : $\operatorname{Inf}(x, y)=\operatorname{Inf}(F)$ et $\operatorname{Sup}(x, y)$ $=\operatorname{Sup}(\mathrm{F})$.

(Le symbole $\square$ désigne la relation d'incomparabilité).

DÉFINITION : Fuseau moyennable

On appelle Fuseau moyennable, un fuseau muni d'une loi de composition interne, de sorte que les tables de composition de toutes les chaînes couvrantes maximales du fuseau soient des carrés moyennables.

LEMME 3.1: Dans un fuseau moyennable $(\mathrm{F}, *, \leq)$, non réduit à une chaîne, l'infimum $\operatorname{Inf}(\mathrm{F})$ et le supremum $\operatorname{Sup}(\mathrm{F})$ sont nécessairement des éléments idempotents ; c'est-àdire $: \operatorname{Inf}(\mathrm{F}) * \operatorname{Inf}(\mathrm{F})=\operatorname{Inf}(\mathrm{F})$ et $\operatorname{Sup}(\mathrm{F}) * \operatorname{Sup}(\mathrm{F})=\operatorname{Sup}(\mathrm{F})$. 
Preuve : (Pour $\operatorname{Inf}(\mathrm{F}))$ Par l'absurde, il existe $\mathrm{x}$ appartenant à une chaîne couvrante maximale tel que $\mathrm{x} ! \neq ! \operatorname{Inf}(\mathrm{F})$ et $\operatorname{Inf}(\mathrm{F}) ! * ! \operatorname{Inf}(\mathrm{F})=\mathrm{x}$. Considérons une autre chaîne ne contenant pas $\mathrm{x}$ (elle existe par hypothèse). Comme elle contient nécessairement $\operatorname{Inf}(\mathrm{F})$, elle n'est pas stable par * puisque $\operatorname{Inf}(\mathrm{F}) * \operatorname{Inf}(\mathrm{F})$ n'appartient pas à cette chaîne ; donc la table de cette chaîne n'est pas un carré moyennable, d'où la contradiction.

PROPOSITION 3.7 : Tout fuseau moyennable $(\mathrm{F}, *, \leq)$ est moyennable.

Preuve : Soit $(\mathrm{F}, *, \leq)$ un fuseau moyennable. Il est clair que la loi $*$ est idemfotente en vertu de ce que toutes les tables de composition de toutes les chaînes couvrantes maximales de $\mathrm{F}$ sont des carrés moyennables. Pour la même raison, on a pour tout $\mathrm{x}$ et y d'une même chaîne $\square: \operatorname{Inf}(\mathrm{x}, \mathrm{y}) \square \square^{\square 1}(\mathrm{x} \square \mathrm{y}) \square \operatorname{Sup}(\mathrm{x}, \mathrm{y})$, avec $\square$ une permutation de ○. Soient, maintenant, deux éléments distincts de deux chaînes distinctes ; ils sont donc incomparables et $\operatorname{Inf}(\mathrm{x}, \mathrm{y})=\operatorname{Inf}(\mathrm{F})$ et $\operatorname{Sup}(\mathrm{x}, \mathrm{y})=\operatorname{Sup}(\mathrm{F})$. Comme la loi $*$ est partout définie, il existe une chaîne $\square$ telle que $\mathrm{x} \square \mathrm{y} \square \square$. Par conséquent, il existe aussi une permutation $\square$ de $\square$ telle que $\square^{\square 1}(\mathrm{x} \square \mathrm{y}) \square \square$ avec $\operatorname{Inf}(\mathrm{F}) \square \square^{\square 1}(\mathrm{x} \square \mathrm{y}) \square \operatorname{Sup}(\mathrm{F})$.

DÉFINITION : Corde à nœuds

On appelle corde à nœuds, un ensemble (E,s) ordonné de sorte qu'il existe une suite finie de fuseaux $F_{1}, F_{2}, \cdots, F_{n}$, vérifiant :

1. $\mathrm{E}=\bigcup_{i=1}^{i=n} \mathrm{~F}_{i}$

2. pour tout entier i de 1 à $n-1$, on a: $F_{i} \square F_{i+1}=\left\{\operatorname{Sup}\left(F_{i}\right)\right\}=\left\{\operatorname{Inf}\left(F_{i+1}\right)\right\}$. L'élément du singleton $F_{i} \square F_{i+1}$ est appelé nœud.

3. pour tout entier $\mathrm{i}$, un des deux fuseaux $\mathrm{F}_{\mathrm{i}}$ ou $\mathrm{F}_{\mathrm{i}+1}$ n'est pas réduit à une seule chaîne.

Remarque : Dans l'ensemble des parties d'un ensemble ordonné (E, $\leq)$, on peut définir l'ordre strict (relation asymétrique et transitive), noté , par : pour tout $\mathrm{A} \square \mathrm{E}$ et tout $\mathrm{B} ! \square \mathrm{E}, \mathrm{A} \quad \mathrm{B}$ si et seulement si pour tout $\mathrm{x} \square \mathrm{A}$ et $\mathrm{y} \square \mathrm{B}$, on a $\mathrm{x} \square \mathrm{y}$. La deuxième condition de la définition permet alors de ranger les fuseaux de $\mathrm{E}$ de sorte que $\mathrm{si} i<\mathrm{j}$, alors $F_{i} \quad F_{j}$.

DÉFINITION : Corde à nœuds moyennable

On appelle corde à nœuds moyennable, une corde à nœuds muni d'une loi de composition interne $*$ vérifiant les conditions suivantes :

1. tous les fuseaux sont moyennables.

2. pour tout entier $\mathrm{i}$ de 1 à $\mathrm{n}-1$, on $\mathrm{a}: \mathrm{si} \mathrm{x} \square \mathrm{F}_{\mathrm{i}}$ et $\mathrm{y} \square \mathrm{F}_{\mathrm{i}+1} \square\left\{\operatorname{Sup}\left(\mathrm{F}_{\mathrm{i}}\right)\right\}$, alors $\mathrm{x}$ !* $\mathrm{y}$ est le nœud de $\mathrm{F}_{\mathrm{i}} \square \mathrm{F}_{\mathrm{i}+1}=\left\{\operatorname{Sup}\left(\mathrm{F}_{\mathrm{i}}\right)\right\}=\left\{\operatorname{Inf}\left(\mathrm{F}_{\mathrm{i}+1}\right)\right\}$.

3. pour tout entier $\mathrm{i}$ de 1 à $\mathrm{n}-2$ et pour tout $\mathrm{j}>\mathrm{i}$, s'il existe au moins un entier $\mathrm{k}$ tel que $\mathrm{i}<\mathrm{k}<\mathrm{j}$, alors pour $\mathrm{x} \square \mathrm{F}_{\mathrm{i}}$ et $\mathrm{y} \square \mathrm{F}_{\mathrm{j}}$, on $\mathrm{a}: \mathrm{x} * \mathrm{y} \square \mathrm{F}_{\mathrm{k}}$. 
PROPOSITION 3.8 : Toute corde à nœuds moyennable $(\mathrm{E}, *, \leq)$ est moyennable.

Preuve : La loi est évidemment idemfotente puisque tous les fuseaux sont moyennables. Reste à examiner la règle de la valeur intermédiaire.

Si x et y appartiennent à $F_{i}$, c'est clair.

Si $x \square F_{i}$ et $y \square F_{i+1} \square\left\{\operatorname{Sup}\left(F_{i}\right)\right\}$, alors $x * y=\operatorname{Sup}\left(F_{1}\right)$ qui est un élément idempotent en vertu du lemme 3.1. Or, pour tout $\mathrm{z} \square \mathrm{F}_{\mathrm{i}}$ et $\mathrm{t} \square \mathrm{F}_{\mathrm{i}+1}$ on a $\mathrm{z} \square \mathrm{t}$ puisque $\operatorname{Sup}\left(\mathrm{F}_{1}\right)=\operatorname{Inf}\left(\mathrm{F}_{\mathrm{i}+1}\right)$; donc en particulier $\mathrm{x} \square \mathrm{y}$. D'où,

$$
\mathrm{X}=\operatorname{Inf}(\mathrm{x}, \mathrm{y}) \square \operatorname{Sup}\left(\mathrm{F}_{1}\right)=\mathrm{m}(\mathrm{x}, \mathrm{y}) \square \operatorname{Sup}(\mathrm{x}, \mathrm{y}=\mathrm{y} .
$$

Si $x \square F_{i}$ et $y \square F_{j}$, et s'il existe $\mathrm{k}$ tel que $\mathrm{i}<\mathrm{k}<\mathrm{j}$, alors on $\mathrm{a}: \mathrm{x} * \mathrm{y} \square \mathrm{F}_{\mathrm{k}}$. Comme $\mathrm{F}_{\mathrm{k}}$ est moyennable, il existe une bijection $\square$ de $\mathrm{F}_{\mathrm{k}}$ telle que $\operatorname{Inf}\left(\mathrm{F}_{\mathrm{k}}\right) \square \square^{\square 1}(\mathrm{x} \square \mathrm{y}) \square \operatorname{Sup}\left(\mathrm{F}_{\mathrm{k}}\right)$. Or, pour $\mathrm{z} \square \mathrm{F}_{\mathrm{k}}$ on $\mathrm{a}: x \square \operatorname{Sup}\left(\mathrm{F}_{1}\right) \square \mathrm{z} \square \operatorname{Inf}\left(\mathrm{F}_{\mathrm{j}}\right) \square y$, donc en particulier pour $\mathrm{z}=\square^{\square 1}(\mathrm{x} \square \mathrm{y})$. Finalement,

$$
\mathrm{x}=\operatorname{Inf}(\mathrm{x}, \mathrm{y}) \square \square^{\square 1}(\mathrm{x} \square \mathrm{y}) \square \mathrm{y}=\operatorname{Sup}(\mathrm{x}, \mathrm{y}) .
$$

Remarque : On peut naturellement continuer le processus de la construction en définissant des «super-fuseaux» - assemblages de cordes maintenues aux extrémités par deux nœuds - puis des «super-cordes» - assemblages de super-fuseaux - et ainsi de suite.

\subsection{Transport de structures moyennables}

Disposant de deux structures moyennables, une première interrogation s'impose. À quelle condition peut-on avoir deux structures moyennables $(\mathrm{E}, *, \leq)$ et $(\mathrm{E},, \leq)$, de même moyenne? La réponse est immédiate, en effet :

PROPOSITION 4.1 : Soient deux structures moyennables : (E,*, $\leq$ ) de moyenne $m_{\square}$ et $(\mathrm{E}, \quad, \leq)$ de moyenne $m$. On a : $m_{\square}=m$ si, et seulement si, il existe une bijection $\mathrm{h}$ de $E$ telle que $a b=h(a \quad b)$.

Preuve : ( $\square$ ) Soit $m_{\square}=m$. Les lois $*$ et sont idemfotentes d'après le théorème!1 (\$!2.3-c). Soient, respectivement, $\mathrm{f}$ et $\mathrm{g}$ les bijections de $\mathrm{E}$ associées. On a : $\mathrm{f}^{\square 1}(\mathrm{a} \square \mathrm{b})=\mathrm{g}^{\square 1}(\mathrm{a} \quad \mathrm{b})$, d'où $\mathrm{a} \square \mathrm{b}=\mathrm{f} \circ \mathrm{g}^{\square 1}(\mathrm{a} \quad \mathrm{b})$ et en posant $\mathrm{h}=\mathrm{f} \circ \mathrm{g}^{\square 1}$, on a bien $\mathrm{h}$ bijective par composition.

( $\square$ ) Soit $h$ une bijection de $E$, telle que : $a \square b=h\left(\begin{array}{ll}a & b\end{array}\right)$. Soit $f$ la bijection associée à *. Considérons, alors, l'application $\mathrm{g}$ définie par : $\mathrm{g}=\mathrm{h}^{\square 1} \circ \mathrm{f}$, elle est bijective et l'on $\mathrm{a}: \mathrm{g}^{-1}\left(\begin{array}{ll}\mathrm{a} & \mathrm{b}\end{array}\right)=\mathrm{f}^{-1} \circ \mathrm{h}(\mathrm{a} \quad \mathrm{b})=\mathrm{f}^{-1}(\mathrm{a} \square \mathrm{b})$. Or, on a pour tout $\mathrm{x}$ de $\mathrm{E}$ : $\mathrm{g}(\mathrm{x})=\mathrm{h}^{-1} \circ \mathrm{f}(\mathrm{x})=\mathrm{h}^{-1}(\mathrm{x} \square \mathrm{x})=\mathrm{x} \quad \mathrm{x}$, donc $\mathrm{g}$ est bien la fonction bijective associée à la loi définie dans $\mathrm{E}$; et par conséquent on a bien : $m_{\square}=m$.

Disposant d'une structure moyennable, il est tentant de construire d'autres structures moyennables issues de celle-ci. C'est ainsi que les moyennes, connues en statistique sous le nom de moyennes d'ordre $r$, sont toutes bâties sur le modèle de la moyenne arithmétique. 
Un procédé classique consiste à se donner une application «h» de $\mathrm{E}$ dans $\mathrm{F}$. On crée la structure $\left(\mathrm{F},, \square_{\mathrm{F}}\right)$ en prenant l'image par «h» de la structure $\left(\mathrm{E}, *, \mathrm{D}_{\mathrm{E}}\right)$ de sorte que :

9. $\mathrm{h}$ est une bijection de $\mathrm{E}$ dans $\mathrm{F}$

10. $h$ est isomorphisme de loi : $h(a \square b)=h(a) h(b)$

11. h est un isomorphisme d'ordre ou un anti-isomorphisme d'ordre.

Dans ces conditions, on dit que $\mathrm{h}$ est isomorphisme de transport.

THÉORÈME du transport : Soient $\left(\mathrm{E}, *, \square_{\mathrm{E}}\right)$ une structure moyennable de moyenne $m_{\sqcap}$, et $\left(\mathrm{F},, \mathrm{G}_{\mathrm{F}}\right)$ une structure obtenue par transport à l'aide d'un isomorphisme $\mathrm{h}$ de transport. La structure $\left(\mathrm{F}, \quad, \square_{\mathrm{F}}\right)$ est, alors, moyennable, de moyenne $m$ avec, pour tout a et $b$ élément de $F !: ! m \quad(a, b)=h \circ m_{\square}\left(h^{\square 1}(a), h^{\square 1}(b)\right)$.

Preuve : montrons d'abord que $\left(\mathrm{F}, \square_{\mathrm{F}}\right)$ est bien réticulé. Il suffit de prouver l'existence d'un $\operatorname{Inf}_{\mathrm{F}}$ et d'un $\operatorname{Sup}_{\mathrm{F}}$ dans $\left(\mathrm{F}, \square_{\mathrm{F}}\right)$. Or, si «h» est croissante, en posant pour tout a et $b$ de $E: \operatorname{Sup}_{F}(h(a), h(b))=h\left(\operatorname{Sup}_{E}(a, b)\right)$, il est aisé de voir que $\operatorname{Sup}_{\mathrm{F}}(\mathrm{h}(\mathrm{a}), \mathrm{h}(\mathrm{b}))$ est le plus petit majorant de $\{\mathrm{h}(\mathrm{a}), \mathrm{h}(\mathrm{b})\}$ et, mutatis mutandis pour $\operatorname{Inf}_{\mathrm{F}}$. De même, si «h» est décroissante, on voit que $\mathrm{h}\left(\operatorname{Inf}_{\mathrm{E}}(\mathrm{a}, \mathrm{b})\right)=\operatorname{Sup}_{\mathrm{F}}(\mathrm{h}(\mathrm{a}), \mathrm{h}(\mathrm{b}))$ est aussi le plus petit majorant de $\{\mathrm{h}(\mathrm{a}), \mathrm{h}(\mathrm{b})\}^{5}$. Par transport, nous avons défini la loi « » par : pour tout $a$ et $b$ de $F, a \quad b=h\left[h^{\square 1}(a) \square h^{\square 1}(b)\right]$. Par conséquent, l'application «g» de $\mathrm{F}$ dans $\mathrm{F}$, définie par : pour tout $\mathrm{x}$ de $\mathrm{E}, \mathrm{g}(\mathrm{x})=\mathrm{x} \quad \mathrm{x}=\mathrm{h} \circ \mathrm{f} \circ \mathrm{h}^{\mathrm{Ql}}(\mathrm{x})$, est bijective comme composée de bijections. Donc « » est idemfotente. En outre, «f» est monotone, donc «g» et $\left\langle\mathrm{g}^{-1} \gg\right.$ aussi par composition de fonctions monotones. D'après le théorème $2(\S$ 2.3-c), c'est fini.

Exemples :

1. Considérons la bijection!: $\mathbb{R}_{+}^{*} \square \mathbb{R}_{+}^{*} \mathrm{x} \mapsto \mathrm{h}(\mathrm{x})=(\mathrm{l} / \mathrm{x})^{\mathrm{n}}$ avec «n» entier non nul, et la moyenne arithmétique définie sur $\left(\mathbb{R}_{+}^{*},+, \square\right)$. Soit «» telle que!: a $\quad \mathrm{b}=1 /(\sqrt[\mathrm{n}]{1 / \mathrm{a}}+\sqrt[\mathrm{n}]{1 / \mathrm{b}})^{\mathrm{n}}$, alors $\left(\mathbb{R}_{+}^{*}, \square\right)$ est moyennable de moyenne!: $m(\mathrm{a}, \mathrm{b})=2^{\mathrm{n}} \mathrm{ab} /(\sqrt[\mathrm{n}]{\mathrm{a}}+\sqrt[\mathrm{n}]{\mathrm{b}})^{\mathrm{n}}$. En particulier, lorsque $\mathrm{n}=1$, on récupère la moyenne harmonique.

2. On passe de la moyenne arithmétique dans $\left(\mathbb{R}_{+},+, \leq\right)$à la moyenne géométrique dans $\left(\mathbb{R}_{+}, \square, \leq\right)$ par l'isomorphisme exponentiel.

3. Dans $P(\mathrm{E})$, ensemble des parties de $\mathrm{E}$, intersection et réunion sont deux moyennes isomorphes obtenues par la bijection décroissante qui, à tout ensemble, associe son complémentaire.

\footnotetext{
${ }^{5}$ Lorsque $\mathrm{h}$ et $\mathrm{h}^{-1}$ sont décroissantes, il faut prouver trois choses :

$-\sup _{\mathrm{F}}(\mathrm{h}(\mathrm{a}), \mathrm{h}(\mathrm{b})) \square \mathrm{F}$, oui car $\inf _{\mathrm{E}}(\mathrm{a}, \mathrm{b}) \square \mathrm{E}$

- $h(a)$ et $h(b) \square_{F} h\left(\inf _{E}(a, b)\right)$, oui car $\inf _{E}(a, b) \square_{E}$ a et b

- $\square x \operatorname{EE}\left[(h(a)\right.$ et $h(b)) \square_{F} h(x) \square h\left(\inf _{E}(a, b) \square_{F} h(x)\right]$,

or, c'est exact car : $\square \times \square E\left[x \square_{E}(a\right.$ et $\left.b) \square \times \square_{E} \inf _{E}(a, b)\right]$.
} 


\section{GENERALISATION}

\subsection{Les p-moyennes}

Considérons un magma réticulé $(\mathrm{E}, *, \leq)$, muni d'une loi de composition interne associative, notée $*$; et un entier $\mathrm{p}$ non nul (on peut éventuellement considérer des lois non associatives à condition, naturellement, de préciser le procédé itératif de formation des composés).

DÉFINITION : On appelle p-moyenne selon la loi associative «*», l'application notée $m$ (lorsqu'elle existe) définie de $\mathrm{E}^{\mathrm{p}}$ dans le magma réticulé $(\mathrm{E}, *, \leq)$ par :

$\mathrm{E} \square \mathrm{E} \square \cdots \square \mathrm{E} \quad \mathrm{E}$ $\left(\mathrm{a}_{1}, \mathrm{a}_{2}, \ldots, \mathrm{a}_{\mathrm{p}}\right) \mapsto m\left(\mathrm{a}_{1}, \mathrm{a}_{2}, \ldots, \mathrm{a}_{\mathrm{p}}\right)$

Axiome $1 \quad \operatorname{Inf}\left(\mathrm{a}_{1}, \mathrm{a}_{2}, \ldots, \mathrm{a}_{\mathrm{p}}\right) \square m\left(\mathrm{a}_{1}, \mathrm{a}_{2}, \ldots, \mathrm{a}_{\mathrm{p}}\right) \square \operatorname{Sup}\left(\mathrm{a}_{1}, \mathrm{a}_{2}, \ldots, \mathrm{a}_{\mathrm{p}}\right)$

Axiome $2 \quad m\left(\mathrm{a}_{1}, \mathrm{a}_{2}, \ldots, \mathrm{a}_{\mathrm{p}}\right)$ est l' unique solution de $\mathrm{l}^{\prime}$ équation :

$$
\underbrace{\mathrm{x} \square \mathrm{x} \square \cdots \square \mathrm{x}}_{\mathrm{p} \text { termes }}=\underbrace{\mathrm{a}_{1} \square \mathrm{a}_{2} \square \cdots \square \mathrm{a}_{\mathrm{p}}}_{\mathrm{p} \text { termes }} \text { où } \mathrm{x} \text { désigne } \mathrm{l}^{\prime} \text { inconnue. }
$$

a) Conjuguée de certaines lois non associatives

Naturellement, le recours à des lois associatives semble inévitable. Cependant, certaines lois non associatives se prêtent à une généralisation possible. Ce sont les lois $\oplus$ qui prennent la forme $: a \oplus b !=! h(a)+h(b)$, où $h$ est une bijection de $E$ et + une loi associative. La loi $*$, dite conjuguée de la loi moment $\oplus$ et définie par : $\mathrm{a} \square \mathrm{b}=\mathrm{h}^{\square 1}(\mathrm{a} \oplus \mathrm{b})=\mathrm{h}^{-1}(\mathrm{~h}(\mathrm{a})+\mathrm{h}(\mathrm{b}))$, est alors associative. En effet, comme la conjuguée est obtenue par transport de + , on récupère l'associativité de celle-ci.

De plus, et c'est ce qui fait son intérêt, si $(\mathrm{E}, \oplus, \leq)$ est 2-moyennable alors $(\mathrm{E}, *, \leq)$ est également 2-moyennable et de même moyenne, dès lors que $\mathrm{h}$ conserve la structure d'ordre, c'est-à-dire $h$ et $h^{\square 1}$ simultanément croissantes ou décroissantes.

Avec cette condition sur $\mathrm{h}$, on a même plus généralement : si $(\mathrm{E},+, \leq)$ est pmoyennable, alors $(\mathrm{E}, *, \leq)$ est $\mathrm{p}$-moyennable et

$$
m_{\square}\left(a_{1}, \cdots, a_{p}\right)=h^{\square 1} \circ m_{+}\left(h\left(a_{1}\right), \cdots, h\left(a_{p}\right)\right) .
$$

b) Propriétés des moyennes généralisées.

Les propriétés essentielles des 2-moyennes sont transmissibles sans peine aux pmoyennes. Passons-les en revue rapidement ; on suppose évidemment que la pmoyenne $m$ définie sur la structure $(\mathrm{E}, *, \leq)$ existe. En outre, la loi «*» est désormais supposée associative et commutative. Dans ces conditions la moyenne possède les propriétés suivantes :

1. Idempotente : pour tout «a» élément de $\mathrm{E}$, on $\mathrm{a}: m(\mathrm{a}, \cdots, \mathrm{a})=\mathrm{a}$

2. Surjective : pour tout «a» de E, il existe $\left(\mathrm{a}_{1}, \cdots, \mathrm{a}_{\mathrm{p}}\right) \square \mathrm{E}^{\mathrm{p}}:$ tel que $\mathrm{a}=m\left(\mathrm{a}_{1}, \cdots, \mathrm{a}_{\mathrm{p}}\right)$ 
3. Non injective : il existe $\left(\mathrm{a}_{1}, \cdots, \mathrm{a}_{\mathrm{p}}\right) \neq\left(\mathrm{b}_{1}, \cdots, \mathrm{b}_{\mathrm{p}}\right)$ avec $m\left(\mathrm{a}_{1}, \cdots, \mathrm{a}_{\mathrm{p}}\right)=m\left(\mathrm{~b}_{1}, \cdots, \mathrm{b}_{\mathrm{p}}\right)$

4. Commutativité : pour toute permutation $\square \square \square_{\mathrm{p}}: m\left(\mathrm{a}_{\square(1)}, \ldots, \mathrm{a}_{\square(\mathrm{p})}\right)=m\left(\mathrm{a}_{1}, \ldots, \mathrm{a}_{p}\right)$

5. Translation : pour tout «a» élément de $\mathrm{E}$, on $\mathrm{a}: m\left(\mathrm{a} \square \mathrm{a}_{1}, \cdots, \mathrm{a} \square \mathrm{a}_{\mathrm{p}}\right)=\mathrm{a} \square m\left(\mathrm{a}_{1}, \cdots, \mathrm{a}_{\mathrm{p}}\right)$

6. Échange des moyens : on pose:

$$
\begin{aligned}
& \text { pour tout } \mathrm{a}_{i, j} \text { de } \mathrm{E} !: \prod_{k=1}^{k=} \mathrm{a}_{k, i}=\mathrm{a}_{1, i} \square \mathrm{a}_{2, i} \square \cdots \square \mathrm{a}_{k, i} \square \cdots \square \mathrm{a}_{n, i} \\
& \text { on a!: }\left.m\right|_{k=1} ^{k=n} \mathrm{a}_{k, 1}, \prod_{k=1}^{k=n} \mathrm{a}_{k, 2}, \cdots, \prod_{k=1}^{k=n} \mathrm{a}_{k, p} \square=\prod_{k=1}^{k=n} \mathrm{~m}\left(\mathrm{a}_{k, 1}, \mathrm{a}_{k, 2}, \cdots, \mathrm{a}_{k, p}\right)
\end{aligned}
$$

en particulier!: $m\left(\mathrm{a}_{1} \square \mathrm{b}_{1}, \cdots, \mathrm{a}_{\mathrm{p}} \square \mathrm{b}_{\mathrm{p}}\right)=m\left(\mathrm{a}_{1}, \cdots, \mathrm{a}_{\mathrm{p}}\right) \square m\left(\mathrm{~b}_{1}, \cdots, \mathrm{b}_{\mathrm{p}}\right)$

7. Homothétie : pour $\mathrm{k}$ entier non nul, on $\mathrm{a}: m\left(\mathrm{k} \cdot \mathrm{a}_{1}, \cdots, \mathrm{k} \cdot \mathrm{a}_{\mathrm{p}}\right)=\mathrm{k} \cdot m\left(\mathrm{a}_{1}, \cdots, \mathrm{a}_{\mathrm{p}}\right)$

8. Monotonie : sous l'hypothèse!: $\mathrm{a} \square \mathrm{c} \square \mathrm{b} \square \mathrm{d} \square[\mathrm{a} \square \mathrm{b}$ et $\mathrm{c} \square \mathrm{d}]$

pour tout a, b, c, d de E ; la moyenne est croissante, c'est-à-dire :

pour tout entier $i$ de 1 à $\mathrm{p}$, si $\mathrm{a}_{i} \square \mathrm{b}_{i}$ alors $m\left(\mathrm{a}_{1}, \cdots \mathrm{a}_{p}\right) \square m\left(\mathrm{~b}_{1}, \cdots \mathrm{b}_{p}\right)$

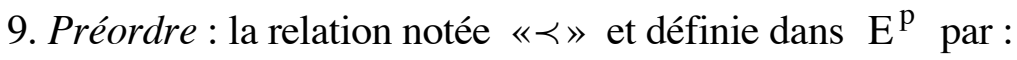

$$
\left(\mathrm{a}_{1}, \ldots, \mathrm{a}_{\mathrm{p}}\right) \prec\left(\mathrm{b}_{1}, \ldots, \mathrm{b}_{\mathrm{p}}\right) \square m\left(\mathrm{a}_{1}, \ldots, \mathrm{a}_{\mathrm{p}}\right) \square m\left(\mathrm{~b}_{1}, \ldots, \mathrm{b}_{\mathrm{p}}\right)
$$

est une relation de préordre. Elle est réflexive, transitive mais n'est pas antisymétrique ; car $m$ n'est pas injective.

c) Conditions de «moyennabilité».

Ce qui a été dit pour les 2-moyennes reste évidemment valable pour les p-moyennes. Il suffit, pour cela, d'adopter la définition suivante : la loi «*» est dite p-idemfotente si et seulement si l'application «f» définie par: $f(x)=p \cdot x$, pour tout «x» de E, est bijective.

Remarquons que si «*» est surjective alors l'application qui, à $\left(a_{1}, \ldots, a_{p}\right)$ associe $\mathrm{a}_{1} \square \ldots \square \mathrm{a}_{\mathrm{p}}$ est elle-même surjective.

Évidemment, lorsque l'ordre est compatible, la bijection «f» associée à la loi p-idemfotente est croissante, et si sa réciproque l'est aussi, alors la moyenne aussi.

De même, lorsque l'ordre est anticompatible, la bijection «f» associée à la loi p-idemfotente est croissante si «p» est impair et décroissante sinon.

Enfin, pour p entier non nul, si $\mathrm{p} \cdot \mathrm{a} \square \mathrm{p} \cdot \mathrm{b} \square$ a $\square \mathrm{b}$ (respectivement $\mathrm{b} \square \mathrm{a}$ ), - en particulier si $(\mathrm{E}, *, \leq)$ est compatible (respectivement anti-compatible) et $\mathrm{E}$ fini ou totalement ordonné -, alors si $(\mathrm{E}, *, \leq)$ est moyennable, il est aussi $\mathrm{p}^{\mathrm{n}}$-moyennable pour tout entier n.

\subsection{Les p-moyennes pondérées}

a) Définition

Considérons un ensemble $\square$ contenant $\mathbb{N}$, de sorte que l'on puisse le munir d'une addition qui prolonge celle de $\mathbb{N}$. Dans ce cas, l'ordre total défini sur $\square$ est archimédien 
pour l'addition, c'est-à-dire!: pour tout $\square$ et $\square$ de $\square$, avec $\square>1$, il existe un entier non nul tel que $\underbrace{\square+\cdots+\square}_{\text {p fois }}>\square$.

On définit, alors, une loi externe, notée «!», sur le magma réticulé $(\mathrm{E}, *, \square)$ en prenant $\square$ pour opérateur.

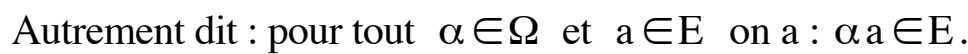

On exige, de cette loi externe, qu'elle vérifie les conditions suivantes :

1. pour tout $\mathrm{x} \square \mathrm{E} 1 \mathrm{x}=\mathrm{x}$

2. pour tout $(\square, \square, \mathrm{x}) \square \square^{2} \square \mathrm{E} \quad(\square+\square) \mathrm{x}=\square \mathrm{x} \square \square \mathrm{x}$

3. pour tout $(\square, \mathrm{x}, \mathrm{y}) \square \square \square \mathrm{E}^{2} \quad \square \mathrm{x}=\square \mathrm{y} \square \mathrm{x}=\mathrm{y}$

4. pour tout $(\square, \mathrm{a}, \mathrm{b}) \square \square \square \mathrm{E}^{2} \quad \square(\mathrm{a} \square \mathrm{b})=\square \mathrm{a} \square \square \mathrm{b}$

DÉFINITION : On appelle p-moyenne pondérée dans $\square$ (selon la loi «*» supposée associative), toute application notée $\widehat{m}$ de $\mathrm{E}^{\mathrm{p}}$ dans $\mathrm{E}$, vérifiant les deux axiomes :

Axiome 1. Pour tout $\square_{i} \square \square$, avec $\square_{i=1}^{i=\mathrm{p}} \square_{\mathrm{i}} \neq 0$ et pour tout $\mathrm{a}_{i} \square \mathrm{E}$.

L'équation : $\square_{1} \mathrm{x} \square \cdots \square \square_{\mathrm{p}} \mathrm{x}=\square_{1} \mathrm{a}_{1} \square \cdots \square \square_{\mathrm{p}} \mathrm{a}_{\mathrm{p}}$ admet une unique solution dans E, notée!: $\hat{m}\left(\square_{1} a_{1}, \cdots, \square_{p} a_{p}\right)$ et appelée moyenne de $\left(a_{1}, \cdots, a_{p}\right)$ pondérée respectivement par $\left(\square_{1}, \cdots, \square_{\mathrm{p}}\right)$.

Axiome 2. $\operatorname{Inf}\left(\mathrm{a}_{1}, \cdots, \mathrm{a}_{\mathrm{p}}\right) \square \hat{m}\left(\square_{1} \mathrm{a}_{1}, \cdots, \square_{\mathrm{p}} \mathrm{a}_{\mathrm{p}}\right) \square \operatorname{Sup}\left(\mathrm{a}_{1}, \cdots, \mathrm{a}_{\mathrm{p}}\right)$.

b) Propriétés

On suppose que la structure $(\mathrm{E}, *, \square)$ admet une p-moyenne pondérée. D'après les axiomes précédents, on établit les propriétés suivantes :

THÉORÈME de correspondance

Pour tout $\square_{i} \square \square$, avec $\square_{i=1}^{i=\mathrm{p}} \square_{\mathrm{i}} \neq 0$, pour tout $\mathrm{a}_{i} \square \mathrm{E}$, et pour tout entier $k_{i}$ non nul, on a :

1. Idempotence : $\hat{m}\left(\square_{1} \mathrm{a}, \cdots, \square_{\mathrm{p}} \mathrm{a}\right)=\mathrm{a}$

2. Uniformité : $\hat{m}\left(\square \mathrm{a}_{1}, \cdots, \square \mathrm{a}_{\mathrm{p}}\right)=m\left(\mathrm{a}_{1}, \cdots, \mathrm{a}_{\mathrm{p}}\right)$

3. Correspondance entière : $\hat{m}\left(\mathrm{k}_{1} \mathrm{a}_{1}, \cdots, \mathrm{k}_{\mathrm{p}} \mathrm{a}_{\mathrm{p}}\right)=m(\underbrace{\mathrm{a}_{1}, \cdots, \mathrm{a}_{1}}_{\mathrm{k}_{1}}, \cdots, \underbrace{\mathrm{a}_{\mathrm{p}} \cdots \mathrm{a}_{\mathrm{p}}}_{\mathrm{k}_{\mathrm{p}}})$

4. Correspondance : $\left(\stackrel{i=\mathrm{p}}{\square} \square_{i=1}\right) \hat{m}\left(\square_{1} \mathrm{a}_{1}, \cdots, \square_{\mathrm{p}} \mathrm{a}_{\mathrm{p}}\right)=\mathrm{p} m\left(\square_{1} \mathrm{a}_{1}, \cdots, \square_{\mathrm{p}} \mathrm{a}_{\mathrm{p}}\right)$

Preuve !: Pour (4) seulement, car (1), (2), et (3) sont des cas particuliers. 
Soit $\hat{m}\left(\square_{1} \mathrm{a}_{1}, \cdots, \square_{\mathrm{p}} \mathrm{a}_{\mathrm{p}}\right)$ l'unique solution de l'équation : $\square_{1} \mathrm{x} \square \cdots \square \square_{\mathrm{p}} \mathrm{x}=\square_{1} \mathrm{a}_{1} \square \cdots \square \square_{\mathrm{p}} \mathrm{a}_{\mathrm{p}}$. D'où $\square_{1} \hat{m}\left(\square_{1} \mathrm{a}_{1}, \cdots, \square_{\mathrm{p}} \mathrm{a}_{\mathrm{p}}\right) \square \cdots \square \square_{p} \hat{m}\left(\square_{1} \mathrm{a}_{1}, \cdots, \square_{\mathrm{p}} \mathrm{a}_{\mathrm{p}}\right)=\square_{1} \mathrm{a}_{1} \square \cdots \square \square_{\mathrm{p}} \mathrm{a}_{\mathrm{p}}$ et d'après la condition 2, à savoir!: $(\square+\square) \mathrm{x}=\square \mathrm{x} \square \square \mathrm{x}$, on en déduit que!: $\left(\square_{i=1}^{i=\mathrm{p}} \square_{i}\right) \hat{m}\left(\square_{1} \mathrm{a}_{1}, \cdots, \square_{\mathrm{p}} \mathrm{a}_{\mathrm{p}}\right)=\square_{1} \mathrm{a}_{1} \square \cdots \square \square_{\mathrm{p}} \mathrm{a}_{\mathrm{p}}$. D'autre part, pour tout entier $i$ non nul, on a $\square_{i} \mathrm{a}_{i} \square \mathrm{E}$, donc $m\left(\square_{1} \mathrm{a}_{1}, \cdots, \square_{\mathrm{p}} \mathrm{a}_{\mathrm{p}}\right)$ est l'unique solution de l'équation!: $x \square \cdots \square x=\square_{1} a_{1} \square \cdots \square \square_{p} a_{p}$. Par conséquent, $p \cdot m\left(\square_{1} a_{1}, \cdots, \square_{p} a_{p}\right)=\square_{1} a_{1} \square \cdots \square \square_{p} a_{p}$. Enfin, comme pour tout $\mathrm{x}$ de $\mathrm{E}, \mathrm{p} \cdot \mathrm{x}=\mathrm{px}$, puisque $\mathbb{N} \square \square$, on a le résultat.

Naturellement, si la structure $(\mathrm{E}, *, \square)$ admet une p-moyenne pondérée dans $\square$, elle admet une p-moyenne (pondération uniforme $\square_{i}=1$ ).

Pour que la réciproque ait lieu, il suffit d'enrichir légèrement la structure $\square$, de sorte que, d'une part, le couple (E, $\square$ ) soit maintenant très proche de la définition d'un espace vectoriel sur un corps $(\square,+, \square)$ - la différence vient de ce que ni $(\mathrm{E}, *)$, ni $(\square,+)$ ne sont supposés présenter une structure de groupe abélien - et d'autre part admettre la compatibilité de la relation d'ordre $\square$ définie sur $\mathrm{E}$ avec les deux lois de composition, interne et externe de $(\mathrm{E}, *, « !))$. Précisons cela.

THÉORÈME de pondération

Soit $(\mathrm{E}, *, \square)$ un magma associatif, réticulé et compatible.

Soit $(\square,+, \square)$ une structure qui opère dans $(\mathrm{E}, *, \ll ! »)$ par le biais d'une loi externe définie sur le couple $(\mathrm{E}, \square)$, «!»: $\square \square \mathrm{E} \square \mathrm{E}$

Le couple $(\mathrm{E}, \square)$ vérifie les conditions suivantes :

1. La structure $(\square,+)$ prolonge celle de $(\mathbb{N},+)$.

2. $(\square-\{0\}, \square)$ est une structure de groupe.

3. Pour tout $\mathrm{x} \square \mathrm{E} 1 \mathrm{x}=\mathrm{x}$ où 1 est l'élément neutre de $(\square-\{0\}$, $\square)$.

4. Pour tout $(\square, \square, \mathrm{x}) \square \square^{2} \square \mathrm{E} \quad(\square+\square) \mathrm{x}=\square \mathrm{x} \square \square \mathrm{x}$.

5. Pour tout $(\square, \square, \mathrm{x}) \square \square^{2} \square \mathrm{E} \quad(\square \square \square) \mathrm{x}=\square(\square \mathrm{x})$.

6. Pour tout $(\square, \mathrm{a}, \mathrm{b}) \square \square \square \mathrm{E}^{2} \quad \square(\mathrm{a} \square \mathrm{b})=\square \mathrm{a} \square \square \mathrm{b}$.

7. Pour tout $(\square, \mathrm{a}, \mathrm{b}) \square \square \square \mathrm{E}^{2}$ a $\square \mathrm{b} \square \square \mathrm{a} \square \square \mathrm{b}$.

Si $(\mathrm{E}, *, \square)$ est $\mathrm{p}$-moyennable, alors il admet une p-moyenne pondérée par toute $\mathrm{p}$ suite $\left(\square_{1}, \square_{2}, \cdots, \square_{p}\right)$ d'éléments de $\square$, telle que $\square_{i=1}^{i=p} \square_{i} \neq 0$.

Preuve : Considérons l'équation : $\square_{1} \mathrm{x} \square \cdots \square \square_{\mathrm{p}} \mathrm{x}=\square_{1} \mathrm{a}_{1} \square \cdots \square \square_{\mathrm{p}} \mathrm{a}_{\mathrm{p}}$. Puisque $(\mathrm{E}, *, \square)$ est p-moyennable et en vertu de la condition 4, on a!: $\bigoplus_{i=1}^{i=p} \square_{i} \square_{\mathrm{x}}=\mathrm{p} m\left(\square_{1} \mathrm{a}_{1}, \cdots, \square_{\mathrm{p}} \mathrm{a}_{\mathrm{p}}\right)$. Comme $\prod_{i=1}^{i=p} \square_{i}$ est supposé non nul, il existe $\square \square \square$ tel que $\square \square \stackrel{i=p}{\square_{i=1}} \square_{i}=1 ;$ d'où, 
puisque la loi externe est une application, on a!: $\square\left(\bigoplus_{i=1}^{i=p} \square_{i} \square_{\square}\right)=\square\left(p m\left(\square_{1} a_{1}, \cdots, \square_{p} a_{p}\right)\right)$.

Mais d'après les conditions 3 et 5 , on a aussi!: $x=\square\left(p m\left(\square_{1} a_{1}, \cdots, \square_{p} a_{p}\right)\right)$ qui est l'unique solution de l'équation caractéristique.

Prouvons, maintenant, que $\operatorname{Inf}\left(\mathrm{a}_{1}, \cdots, \mathrm{a}_{\mathrm{p}}\right) \square \square\left(\mathrm{p} m\left(\square_{1} \mathrm{a}_{1}, \cdots, \square_{\mathrm{p}} \mathrm{a}_{\mathrm{p}}\right)\right) \square \operatorname{Sup}\left(\mathrm{a}_{1}, \cdots, \mathrm{a}_{\mathrm{p}}\right)$. Comme pour tout $\mathrm{a}_{i} \square \mathrm{E}$ on a $\operatorname{Inf}\left(\mathrm{a}_{1}, \cdots, \mathrm{a}_{\mathrm{p}}\right) \square \mathrm{a}_{i}$ et $\mathrm{a}_{i} \square \operatorname{Sup}\left(\mathrm{a}_{1}, \cdots, \mathrm{a}_{\mathrm{p}}\right)$, par la condition 6 on a pour tout $\square_{i} \square \square, \square_{i} \operatorname{Inf}\left(\mathrm{a}_{1}, \cdots, \mathrm{a}_{\mathrm{p}}\right) \square \square_{i} \mathrm{a}_{i}$ et $\square_{i} \mathrm{a}_{i} \square \square_{i} \operatorname{Sup}\left(\mathrm{a}_{1}, \cdots, \mathrm{a}_{\mathrm{p}}\right)$. Par compatibilité de $\square$ et de $*$ on obtient : $\left(\underset{i=1}{i=\mathrm{p}} \square_{i}\right) \operatorname{Inf}\left(\mathrm{a}_{1}, \cdots, \mathrm{a}_{\mathrm{p}}\right) \square \square_{1} \mathrm{a}_{1} \square \cdots \square \square_{\mathrm{p}} \mathrm{a}_{\mathrm{p}}$ c'est-à-dire encore $\left(\square_{i=1}^{i=\mathrm{p}} \square_{i}\right) \operatorname{Inf}\left(\mathrm{a}_{1}, \cdots, \mathrm{a}_{\mathrm{p}}\right) \square{ }_{i=1}^{\mathrm{i}=p} \square_{i} \mathrm{~B}_{\mathrm{x}}$ et finalement d'après les conditions 2, 3, 5 et 7, on obtient!: $\operatorname{Inf}\left(\mathrm{a}_{1}, \cdots, \mathrm{a}_{\mathrm{p}}\right) \square \mathrm{x}$; et mutatis mutandis pour la borne Sup. Ainsi, la p-moyenne pondérée existe et, en la notant $\hat{m}$, nous pouvons poser!: $\bar{m}\left(\square_{1} \mathrm{a}_{1}, \cdots, \square_{\mathrm{p}} \mathrm{a}_{\mathrm{p}}\right)$ $=\square\left(\mathrm{p} m\left(\square_{1} \mathrm{a}_{1}, \cdots, \square_{\mathrm{p}} \mathrm{a}_{\mathrm{p}}\right)\right)$ avec $\square \square \square$ tel que $\square \square \stackrel{i=p}{i=1} \square_{i}=1$.

\subsection{Propriétés barycentriques}

Les moyennes les plus intéressantes sont généralement construites à partir de lois régulières et ne sont donc pas des opérations associatives (au sens usuel du terme). En revanche, toutes les moyennes, sans exception, jouissent d'une propriété d'associativité particulière, connue sous le nom de associativité barycentrique. Cette propriété découle d'une autre, plus élémentaire encore, et qui dit en substance ceci : la moyenne d'une liste de «p» éléments reste inchangée lorsque l'on substitue «q» éléments ( $\mathrm{q} \sqcap \mathrm{p})$ de cette liste par la moyenne de ces «q» éléments, sous réserve bien sûr que cette moyenne existe. Plus précisément :

THÉORÈME de la moyenne partielle

Soit un magma $(\mathrm{E}, *, \square)$ « » et «q» moyennable avec $(\mathrm{q} \sqcap \mathrm{p})$, alors : pour tout $i \square \mathbb{N}^{*}$ tel que $1 \square i \square \mathrm{p}$, on a :

$$
\mathrm{m}\left(\mathrm{a}_{1}, \cdots, \mathrm{a}_{\mathrm{p}}\right)=\mathrm{m}\left(\mathrm{a}_{1}, \cdots, \mathrm{a}_{i}, \mathrm{~m}\left(\mathrm{a}_{i+1}, \cdots, \mathrm{a}_{i+\mathrm{q}}\right), \cdots, \mathrm{m}\left(\mathrm{a}_{i+1}, \cdots, \mathrm{a}_{i+\mathrm{q}}\right), \mathrm{a}_{i+\mathrm{q}+1}, \cdots \mathrm{a}_{\mathrm{p}}\right)
$$

Comme les notations se comprennent d'elles-mêmes, nous avons utilisé le même symbole pour désigner la p-moyenne et la q-moyenne afin d'éviter toute surcharge d'écriture. Il va de soi que si la loi est commutative, toute permutation conserve l'égalité. De plus et toujours sous réserve que les moyennes existent, il est possible aussi de faire intervenir plusieurs moyennes partielles distinctes comme dans l'exemple suivant!: $m(\mathrm{a}$, $\mathrm{b}, \mathrm{c}, \mathrm{d}, \mathrm{e}, \mathrm{f})=m(m(\mathrm{a}, \mathrm{b}), m(\mathrm{a}, \mathrm{b}), \mathrm{c}, m(\mathrm{~d}, \mathrm{e}, \mathrm{f}), m(\mathrm{~d}, \mathrm{e}, \mathrm{f}), m(\mathrm{~d}, \mathrm{e}, \mathrm{f}))$.

On comprend dès lors l'intérêt pratique (et théorique) de la moyenne pondérée ; elle nous permet d'exploiter commodément la propriété des moyennes partielles. Ainsi selon l'exemple cité plus haut, nous avons : $m(\mathrm{a}, \mathrm{b}, \mathrm{c}, \mathrm{d}, \mathrm{e}, \mathrm{f})=\widehat{m}(2 m(\mathrm{a}, \mathrm{b}), \mathrm{c}, 3 m(\mathrm{~d}, \mathrm{e}, \mathrm{f}))$. Toutes ces remarques nous conduisent aux deux théorèmes suivants, valables même si la pondération n'est pas entière. 
THÉORÈME de l'associativité barycentrique

Soit un magma $(\mathrm{E}, *, \square)$ «p et «q» moyennable avec $(\mathrm{q} \sqcap \mathrm{p})$, alors : pour tout $i \square \mathbb{N}^{*}$ tel que $1 \square i \square \mathrm{p}$, on a :

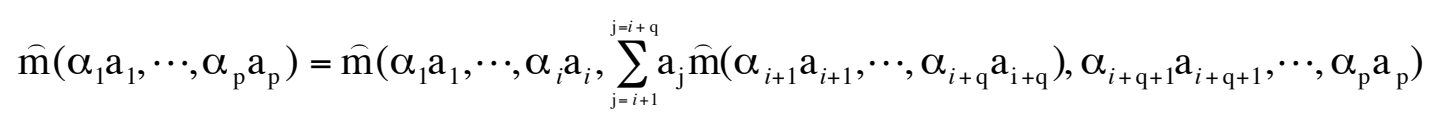

Exemple : $\widehat{m}(\mathrm{a}, 3 \mathrm{~b}, \mathrm{c}, 5 \mathrm{~d}, 7 \mathrm{e})=\hat{m}(\mathrm{a}, 9 \widehat{m}(3 \mathrm{~b}, \mathrm{c}, 5 \mathrm{~d}), 7 \mathrm{e})$

THÉORÈME des moyennes emboîtées

Soit un magma $(\mathrm{E}, *, \square)$ pondérable et moyennable à tout ordre, on a :

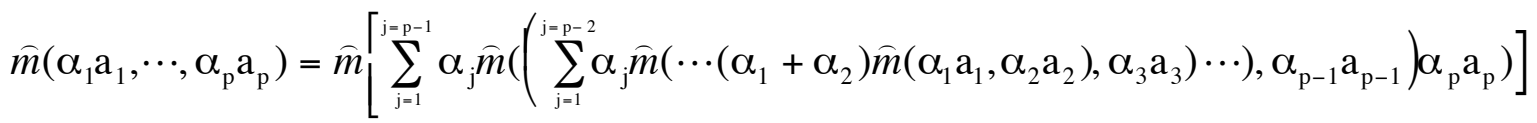

Exemple : $\hat{m}(\mathrm{a}, 3 \mathrm{~b}, \mathrm{c}, 5 \mathrm{~d}, 7 \mathrm{e})=\widehat{m}(10 \hat{m}(5 \hat{m}(4 \hat{m}(\mathrm{a}, 3 \mathrm{~b}), \mathrm{c}), 5 \mathrm{~d}), 7 \mathrm{e})$.

\section{CONCLUSION}

Les ensembles numériques infinis, dénombrables ou non, qui se prêtent le plus volontiers à une généralisation de la moyenne arithmétique sont ceux qui sont ordonnés selon un ordre divisible (ordre total pour lequel aucun intervalle à bornes distinctes n'est vide). Dans la mesure, où, le plus souvent, ils sont munis d'opérations compatibles avec l'ordre, il devient aisé d'établir des conditions de moyennabilité. Mais la compatibilité n'est pas un critère décisif, pour autant. Le cas du corps des complexes est, à ce propos, éloquent. Bien qu'aucun ordre ne soit compatible avec cette structure, il existe néanmoins des ordres pour lesquels la moyenne subsiste. De même, l'exigence d'ordres totaux n'est pas nécessaire non plus, comme le montre, au début de l'article, l'exemple avec les développements décimaux de nombres réels.

Dans le domaine fini, en particulier non numérique, la situation est plus claire. Si l'ordre est total, alors ne sont moyennables que les structures dont la loi de composition vérifie le critère du carré moyennable. Cette exigence appauvrit considérablement les structures concernées; ou bien la loi de composition est idempotente, ou bien elle n'est ni associative, ni compatible avec l'ordre. Dans le premier cas, on retrouve les opérations usuelles définies sur des ensembles finis ; dans le second cas, il devient difficile d'envisager des constructions de moyennes de plus de deux éléments.

\section{BIBLIOGRAPHIE}

[1] ANTOINE, C., Les moyennes, Collection Que sais-je ?, Paris, Presses Universitaires de France, 1998.

[2] BARBUT, M., «Sur une classe de résumés statistiques : les valeurs centrales», L'àpeu-près, Paris, Édition de l'École des Hautes Études en Sciences Sociales, 1988, p.!109141.

[3] BARBUT, M., «Moyennes et valeurs centrales», Moyenne, milieu, centre. Histoires et Usages, Paris, Édition de l'École des Hautes Études en Sciences Sociales, 1991, p.!135151 
[4] BARBUT, M., MONJARDET, B., Ordre et classification, algèbre et combinatoire, Paris, Hachette, 1970, 2 tomes.

[5] HARDY, G., LITTLEWOOD, J. and POLYA, G., Inequalities, Cambridge University Press, 1934.

[6] YULE, G.V., KENDALL, M.G., An introduction to the theory of statistics, Published by Charles Griffin and Co 2td, London, 1958. 